Submitted to the Annals of Probability

\title{
CUTOFF PHENOMENON FOR THE ASYMMETRIC SIMPLE EXCLUSION PROCESS AND THE BIASED CARD SHUFFLING
}

\author{
By Cyril LabBé* ANd Hubert Lacoin ${ }^{\dagger}$ \\ Université Paris-Dauphine* and IMPA ${ }^{\dagger}$
}

\begin{abstract}
We consider the biased card shuffling and the Asymmetric Simple Exclusion Process (ASEP) on the segment. We obtain the asymptotic of their mixing times: our result show that these two continuous-time Markov chains display cutoff. Our analysis combines several ingredients including: a study of the hydrodynamic profile for ASEP, the use of monotonic eigenfunctions, stochastic comparisons and concentration inequalities.
\end{abstract}

\section{CONTENTS}

1 Introduction . . . . . . . . . . . . . . . . . 1

2 Model and results . . . . . . . . . . . . . . . . . 3

3 Technical preliminaries . . . . . . . . . . . . . . . 10

4 Getting the mixing times from scaling limits . . . . . . . . . 18

5 Hydrodynamic limit . . . . . . . . . . . . . . . . . 29

6 Locating the leftmost particle using the hydrodynamic profile . . . 40

A Grand coupling . . . . . . . . . . . . . . 47

References........................... 48

Author's addresses . . . . . . . . . . . . . . . . 50

1. Introduction. The relaxation to equilibrium for particle systems is a subject that has given rise to a rich literature. The phenomenon has been studied from different viewpoints: importance was first given to the problem of the evolution of the particle density on a macroscopic space scale and an adequate time scale, which is usually referred to as hydrodynamic limits (see [17] for a detailed account on the subject as well as references), but the modern theory of Markov chains highlighted another aspect of the problem, which is how the equilibrium state is approached in terms of distance between probability measures, or the mixing time problem [24].

While hydrodynamic limits are now fairly well understood for the simplest particle systems (exclusion process, zero range etc...), there are still

MSC 2010 subject classifications: Primary 60J27; secondary 37A25, 82C22

Keywords and phrases: Card shuffling; Exclusion process; ASEP; Mixing time; Cutoff. 
some fundamental questions on the mixing time that remain unsolved. In particular, it is believed that for many particle systems convergence to equilibrium occurs abruptly, a phenomenon known as cutoff (see [24, Chapter 18] for an introduction to cutoff and examples of Markov chains with cutoff). Until now, this has been rigorously proved to hold only for some specific cases among which the simple (symmetric) exclusion process on the complete graph [12] or in one dimensional graphs (segment and circle) [20, 21].

In many other cases a weaker version of the statement, called pre-cutoff, has been proved (see below for a precise definition). This includes for instance the process which is the focus of this paper: the Asymmetric Simple Exclusion Process (ASEP) on the segment [4] (and also more recently [15]).

The ASEP can be defined as follows: $k$ particles on a segment of length $N$ jump independently with rate $p>1 / 2$ to the right and $q=(1-p)$ to the left. A restriction is added: each site can be occupied by at most one particle, so that every jump which yields a configuration that violates this restriction is canceled. We also study the biased card shuffling which is a walk on the symmetric group from which the ASEP can be obtained as a projection; this Markov chain also displays pre-cutoff.

While it has been known, since a counter example was proposed by Aldous in 2004 (see [24, Figure 18.2]) that it is possible to have pre-cutoff without cutoff, it is a folklore conjecture in the field that all "reasonable" Markov chains with pre-cutoff should in fact have cutoff, thus providing many open problems (see [24, Section 23.2]).

The main achievement of this paper is to show that indeed cutoff holds for the ASEP and to identify the asymptotic behavior for the mixing time. This solves a question which had been left open since the publication of [4]. We prove that the mixing time corresponds exactly to the time at which the particle density stabilizes to the equilibrium profile: this underlines the connection between hydrodynamic limits and mixing times (which was already underlined in the symmetric case see e.g. [21, 22]). We also derive a similar result for the biased card shuffling.

Note that while the hydrodynamic limit for the asymmetric exclusion process on the full line has been well understood for many years [29] (also $[3,26,30]$ for the special "wedge" initial condition), the presence of boundary conditions makes the problem more delicate to analyze, and a substantial part of our paper is devoted to the analysis of the scaling limits of two quantities associated with the ASEP:

- the particle density (which had been analyzed in the small biased case by one of the authors [18]),

- the positions of the leftmost particle and the rightmost empty site 
(which, depending on the initial condition, may or may not coincide with what is suggested by the limit of the particle density).

\section{Model and results.}

2.1. Biased card shuffling. Given $N \in \mathbb{N}$ and $p \in(1 / 2,1]$, we set $q=$ $1-p$ and consider the following continuous time Markov chain on the set of permutations of $N$ cards labeled from one to $N$. Each pair of adjacent cards is chosen at rate one: then, with probability $p$ (corresponding to an independent Bernoulli random variable) we arrange the cards such that the lower card comes before the higher card and with probability $q$ we arrange them so that the higher card comes first.

A configuration of cards can be represented by an element $\sigma$ of the symmetric group $\mathcal{S}_{N}$ : for every $i \in \llbracket 1, N \rrbracket, \sigma(i)$ (we use the notation $\llbracket a, b \rrbracket=$ $[a, b] \cap \mathbb{Z}$ ) denotes the label of the card at position $i$. The dynamics presented above then corresponds to the Markov process on $\mathcal{S}_{N}$ with the following generator:

$$
\begin{aligned}
\mathcal{L}_{N} f(\sigma) & \left.:=\sum_{i=1}^{N-1}\left(p \mathbf{1}_{\{\sigma(i+1)<\sigma(i)\}}+q \mathbf{1}_{\{\sigma(i+1)>\sigma(i)\}}\right)\left[f\left(\sigma \circ \tau_{i}\right)\right)-f(\sigma)\right] \\
& =\sum_{i=1}^{N-1} p\left[f\left(\sigma^{i,+}\right)-f(\sigma)\right]+q\left[f\left(\sigma^{i,-}\right)-f(\sigma)\right] .
\end{aligned}
$$

In the expression above, $\tau_{i}$ denotes the transposition $(i, i+1)$ and $\sigma^{i,+}, \sigma^{i,-}$ denote the elements of $\mathcal{S}_{N}$ which satisfy the following property

$$
\begin{cases}\sigma^{i, \pm}(j) & =\sigma(j), \quad \forall j \in \llbracket 1, N \rrbracket \backslash\{i, i+1\} \\ \sigma^{i,+}(i) & <\sigma^{i,+}(i+1) \\ \sigma^{i,-}(i) & >\sigma^{i,-}(i+1) .\end{cases}
$$

Note that either $\sigma^{i,+}$ or $\sigma^{i,-}$ is equal to $\sigma$ so that the choice of a pair of cards does not always imply a modification of the permutation.

As $p>q$, this way of shuffling cards favors permutations which are more "ordered". More precisely, if we let $D(\sigma)$ denote the minimal number of adjacent transpositions needed to obtain $\sigma$ starting from the identity permutation (the graph distance between $\sigma$ and the identity in the Cayley graph of $\mathcal{S}_{N}$ with generator $\left(\tau_{i}\right)_{i=1}^{N-1}$ ), then one can check that the equilibrium measure is given by

$$
\pi_{N}(\sigma):=\frac{\lambda^{-D(\sigma)}}{\sum_{\sigma^{\prime} \in \mathcal{S}_{N}} \lambda^{-D\left(\sigma^{\prime}\right)}},
$$

imsart-aop ver. 2014/10/16 file: AOP1290.tex date: May 9, 2019 
where $\lambda=p / q$. The detailed balance condition is easy to check with the relation

$$
D(\sigma):=\sum_{i<j} \mathbf{1}_{\{\sigma(i)>\sigma(j)\}} .
$$

In the particular case $p=1$, the parameter $\lambda$ equals $+\infty$ and the equilibrium measure $\pi_{N}$ is a Dirac measure at the identity permutation.

We denote the process starting from initial condition $\xi \in \mathcal{S}_{N}$ by $\sigma_{t}^{\xi}$ and let $Q_{t}^{\xi}$ denote the distribution of $\sigma_{t}^{\xi}$ at time $t$.

Recall that the total variation distance between two probability measures $\alpha$ and $\beta$ on some discrete space $\Omega$ is defined by

$$
\begin{aligned}
\|\alpha-\beta\|_{T V} & :=\frac{1}{2} \sum_{x \in \Omega}|\alpha(x)-\beta(x)|=\max _{A \subset \Omega}[\alpha(A)-\beta(A)] \\
& =\inf _{\substack{X_{1} \sim \alpha \\
X_{2} \sim \beta}} P\left(X_{1} \neq X_{2}\right),
\end{aligned}
$$

where the infimum is taken over all couplings that give distribution $\alpha$ to $X_{1}$ and $\beta$ to $X_{2}$. The fact that the three definitions are equivalent is a standard property see e.g. [24, Section 4.1]. Using standard terminology, we define the (worst-case) total-variation distance to equilibrium by

$$
d^{N}(t):=\max _{\xi \in \mathcal{S}_{N}}\left\|Q_{t}^{\xi}-\pi_{N}\right\|_{T V}
$$

and the corresponding mixing time by

$$
T_{\text {mix }}^{N}(\varepsilon):=\inf \left\{t \geqslant 0: d^{N}(t)<\varepsilon\right\} .
$$

A notion very much related to mixing time is that of cutoff, which designates a form of abrupt convergence to equilibrium for Markov chain. For an arbitrary sequence of Markov chains, cutoff is said to hold if for all $\varepsilon>0$,

$$
\lim _{N \rightarrow \infty} \frac{T_{\mathrm{mix}}^{N}(\varepsilon)-T_{\mathrm{mix}}^{N}(1-\varepsilon)}{T_{\mathrm{mix}}^{N}(1 / 4)}=0 .
$$

If $\sup _{\varepsilon \in(0,1)} \lim \sup _{N \rightarrow \infty}\left(T_{\text {mix }}^{N}(\varepsilon)-T_{\text {mix }}^{N}(1-\varepsilon)\right) / T_{\text {mix }}^{N}(1 / 4)<\infty$ we say that pre-cutoff holds.

We define gap $_{N}$ to be the spectral gap for this Markov chain. Recall that for a continuous time reversible, irreducible Markov chain with generator $\mathcal{L}$ on a finite state space, the spectral gap is simply defined as the smallest positive eigenvalue of $-\mathcal{L}[24$, Section 20.3]. The spectral gap controls the 
asymptotic rate of convergence to equilibrium (see [24, Corollary 12.6]), in the case of our Markov chains this gives

$$
\lim _{t \rightarrow \infty} \frac{1}{t} \log d^{N}(t)=-\operatorname{gap}_{N} .
$$

Our main result is the following

TheOREm 1. We have for every $p \in(1 / 2,1]$ and $\varepsilon \in(0,1)$

$$
\lim _{N \rightarrow \infty} \frac{T_{\mathrm{mix}}^{N}(\varepsilon)}{N}=\frac{2}{p-q} .
$$

Moreover we have for every value of $N$ and $p$

$$
\operatorname{gap}_{N}=(\sqrt{p}-\sqrt{q})^{2}+4 \sqrt{p q} \sin \left(\frac{\pi}{2 N}\right)^{2} .
$$

Let us stress that another proof of (3) provided by Levin and Peres in [25] appeared while we were in the process of writing the present paper.

REMARK 1. Note that gap ${ }_{N}$ coincides with the spectral gap of the biased walk with transition rates $p$ and $q$ on the segment (and this will also be the case for the ASEP). This result is reminiscent of Aldous' spectral gap conjecture, now a theorem proved by Caputo, Liggett and Richthammer [9], which states that the spectral gap for the interchange process on an arbitrary graph equals that of the corresponding random walk. However let us stress that the biased card shuffling is not an interchange process, and that our results can not be deduced from the one in [9].

REMARK 2. Observe that $\operatorname{gap}_{N} T_{\text {mix }}^{N}(\varepsilon) \rightarrow \infty$ as $N \rightarrow \infty$, and recall that this condition is necessary (but not sufficient) for having a pre-cutoff, see [24, Sec 18.3].

Note that intuitively, as the shuffle tends to order the pack, the worst initial condition should be the permutation $\sigma_{\max }$ defined by

$$
\sigma_{\max }: i \mapsto N+1-i
$$

Our proof implies indeed that this is asymptotically the case. 
2.2. Asymmetric Simple Exclusion Process. Given $k \in \llbracket 1, N-1 \rrbracket$, we obtain another Markov process if we decide to follow only the positions of the cards labeled from $N-k+1$ to $N$, that is if we consider the image of $\left(\sigma_{t}\right)_{t \geq 0}$ by the transformation

$$
\sigma \mapsto \mathbf{1}_{\llbracket N-k+1, N \rrbracket} \circ \sigma .
$$

(Our choice of following the particles with higher rather than lower labels may seem unnatural, but is driven by the fact that we want the particles to drift to the right).

It is not difficult to check that the process obtained via this transformation is indeed Markov. A more intuitive description is the following. Consider $k$ particles on the segment $I_{N}:=\llbracket 1, N \rrbracket$ which are initially placed on $k$ distinct sites. The particles perform independent, continuous time, random walks on $I_{N}$ with jump rate $p$ to the right and $q$ to the left (a particle at site 1, resp. $N$, is not allowed to jump to its left, resp. right): however, if a particle tries to jump on an occupied site, the jump is cancelled.

Denoting the presence of particle by 1 s and their absence by 0 s, the space of configurations associated to this process is given by

$$
\Omega_{N, k}^{0}=\left\{\eta \in\{0,1\}^{I_{N}}: \sum_{i=1}^{N} \eta(i)=k\right\} .
$$

We denote the evolving particle system by $\left(\eta^{\xi}(t, \cdot)\right)_{t \geqslant 0}$ where $\eta^{\xi}(t, x)$ equals 1 if there is a particle at site $x$ at time $t$, and 0 otherwise while $\xi$ underlines the dependence on the initial condition. The law of $\eta^{\xi}(t)$ is denoted by $P_{t}^{\xi}$. Now if we set for $\eta \in \Omega_{N, k}^{0}$,

$$
A(\eta):=\left(\sum_{i=1}^{N} \eta(i)(N-i)\right)-\frac{k(k-1)}{2}
$$

then the equilibrium measure $\pi_{N, k}$ for the dynamics is simply the image of $\pi_{N}$ by the transformation (5), namely

$$
\pi_{N, k}(\eta):=\frac{\lambda^{-A(\eta)}}{\sum_{\eta^{\prime} \in \Omega_{N, k}^{0}} \lambda^{-A\left(\eta^{\prime}\right)}} .
$$

We also define the associated distance to equilibrium and mixing time to be respectively

$$
\begin{aligned}
& d^{N, k}(t):=\max _{\xi \in \Omega_{N, k}^{0}}\left\|P_{t}^{\xi}-\pi_{N, k}\right\|_{T V}, \\
& T_{\text {mix }}^{N, k}(\varepsilon):=\inf \left\{t \geqslant 0: d^{N}(t)<\varepsilon\right\} . \\
& \text { imsart-aop ver. } 2014 / 10 / 16 \text { file: AOP1290.tex date: May } 9,2019
\end{aligned}
$$


We are going to compute the mixing time for the system in the limit where $k / N$ tends to $\alpha \in[0,1]$. Even though we allow the values 0 and 1 for $\alpha$, we always impose $k \geq 1$ and $k \leq N-1$ in order to exclude settings where the state-space becomes trivial $\left(\# \Omega_{N, k}^{0}=1\right)$. We use the notation

$$
\lim _{\substack{N \rightarrow \infty \\ k / N \rightarrow \alpha}} J(k, N)=l,
$$

to express that the limit of the real valued function $J(k, N)$ is $l$ for all sequences such that $k / N$ tends to $\alpha$, or in other words

$$
\lim _{\varepsilon \rightarrow 0} \limsup _{N \rightarrow \infty} \sup _{\substack{k \in \llbracket 1, N-1 \rrbracket \\|k / N-\alpha| \leq \varepsilon}}|J(k, N)-l|=0 .
$$

TheOREm 2. We have for every $p \in(1 / 2,1]$, every $\alpha \in[0,1]$ and every $\varepsilon \in(0,1)$

$$
\lim _{\substack{N \rightarrow \infty \\ k / N \rightarrow \alpha}} \frac{T_{\mathrm{mix}}^{N, k}(\varepsilon)}{N}=\frac{(\sqrt{\alpha}+\sqrt{1-\alpha})^{2}}{p-q} .
$$

Moreover for every $N$, every $k \in \llbracket 1, N-1 \rrbracket$ and every $p$ we have

$$
\operatorname{gap}_{N, k}=(\sqrt{p}-\sqrt{q})^{2}+4 \sqrt{p q} \sin \left(\frac{\pi}{2 N}\right)^{2} .
$$

By symmetry, an analogous result holds for the case $p \in[0,1 / 2)$. The behavior of the system for $p=1 / 2$ is very different and was the object of a particular study [20] where it is shown that cutoff holds on the time scale $N^{2} \log N$ confirming a conjecture of Wilson [32].

REMARK 3. We have mentioned that it was sufficient to consider the case $p \in(1 / 2,1]$. Let us also mention that for the proof of Theorem 2, we only need to treat the case $\alpha \leq 1 / 2$ (and in all the paper we apply this restriction). These two facts are consequences of well known symmetry considerations, which we detail here for the sake of completeness.

For the biased card shuffling, we can notice (recall (4)) that given $\xi \in \mathcal{S}_{N}$, $\sigma_{t}^{\xi} \circ \sigma_{\max }$ is a card shuffling with bias $(1-p)$ and initial condition $\xi \circ \sigma_{\max }$ : the distance to equilibrium is left unchanged under permutation of the state space, and therefore the mixing time is invariant upon reversing the bias.

As a consequence, for $\xi \in \Omega_{N, k}^{0}$, the process $\eta_{t}^{\xi} \circ \sigma_{\max }$ is an ASEP with opposite bias, and by the same argument as above, we find that the mixing time is invariant under $p \mapsto 1-p$. Moreover if $\mathbf{1}-\xi$ denotes the configuration where zeros and ones are swapped, then $\mathbf{1}-\xi \in \Omega_{N, N-k}^{0}$ and $\mathbf{1}-\eta_{t}^{\xi}$ is an ASEP with opposite bias and complementary density of particles. Hence, the mixing time is invariant under the map $k \mapsto N-k$. 
2.3. Mixing time from an arbitrary initial condition. Instead of the worstcase total-variation distance to equilibrium, we can also consider the totalvariation distance to equilibrium starting from a given configuration $\xi_{N} \in$ $\Omega_{N, k}^{0}$ :

$$
d^{\xi_{N}}(t):=\left\|P_{t}^{\xi_{N}}-\pi_{N, k}\right\|_{T V},
$$

and the associated notion of mixing time

$$
T_{\text {mix }}^{\xi_{N}}(\varepsilon):=\inf \left\{t \geqslant 0: d^{\xi_{N}}(t)<\varepsilon\right\} .
$$

We will show that asymptotically, the mixing time depends on three characteristics of $\xi_{N}$ : The initial location of the leftmost particle, the initial location of the rightmost empty site, and the initial empirical density of particles (a probability distribution on the interval). We thus introduce the following notation, for $\xi \in \Omega_{N, k}^{0}$ :

$$
\begin{aligned}
& \ell_{N}(\xi)=\min \{x \in \llbracket 1, N \rrbracket: \xi(x)=1\}, \\
& r_{N}(\xi)=\max \{x \in \llbracket 1, N \rrbracket: \xi(x)=0\} .
\end{aligned}
$$

As when properly renormalized, $\ell_{N}, r_{N}$ and the particle density all belong to compact sets, from any sequences $k_{N}$ ( and $\xi_{N}, N \geqslant 1$ ), one can extract a subsequence along which the three quantities converge. Hence, without loss of generality, we will assume (when $k / N \rightarrow \alpha>0$ ) that there exist $\ell, r \in[0,1]$ and $\rho_{0} \in L^{\infty}([0,1])$ such that

$$
\begin{aligned}
& \lim _{N \rightarrow \infty} \frac{\ell_{N}\left(\xi_{N}\right)}{N}=\ell, \quad \lim _{N \rightarrow \infty} \frac{r_{N}\left(\xi_{N}\right)}{N}=r, \\
& \lim _{N \rightarrow \infty} \frac{1}{N} \sum_{x=1}^{N} \xi_{N}(x) \delta_{x / N}(d y)=\rho_{0}(y) \mathrm{d} y .
\end{aligned}
$$

The convergence to $\rho_{0}$ is meant to hold in the weak topology, that is, for any continuous function $\varphi \in \mathcal{C}([0,1])$ we have

$$
\lim _{N \rightarrow \infty} \frac{1}{N} \sum_{x=1}^{N} \xi_{N}(x) \varphi(x / N)=\int_{[0,1]} \rho_{0}(x) \varphi(x) \mathrm{d} x .
$$

Note that we necessarily have $\int \rho_{0}(x) \mathrm{d} x=\alpha$. In the case where the limit of $k / N$ vanishes, we only require convergence for $\ell_{N}$ as the other two are trivial.

We prove that under these conditions and provided that $\ell<r$, the mixing time starting from $\xi_{N}$ also displays cutoff on scale $N$. More precisely, 
TheOREM 3. Assuming that the sequence $\left(\xi_{N}, N \geqslant 1\right)$ of initial conditions satisfies (10), we have for all $\epsilon \in(0,1)$,

$$
\lim _{N \rightarrow \infty} \frac{T_{\mathrm{mix}}^{\xi_{N}}(\varepsilon)}{N}=\frac{1}{p-q} \max \left(t_{\rho_{0}}, 1-\alpha-\ell, r-1+\alpha\right)
$$

where $t_{\rho_{0}}$ is a function of the initial density.

In the case where $k / N \rightarrow 0$ and $\lim _{N \rightarrow \infty} \ell_{N}\left(\xi_{N}\right) / N=\ell$, we have

$$
\lim _{N \rightarrow \infty} \frac{T_{\mathrm{mix}}^{\xi_{N}}(\varepsilon)}{N}=\frac{1-\ell}{p-q}
$$

The definition of $t_{\rho_{0}}$ is given in Section 4.1 where we introduce the scaling limit for the process of empirical densities: it corresponds to the time needed by this scaling limit (the solution of the Burgers equation) to reach its steady state.

REMARK 4. At the cost of introducing some extra notation, we could also state (and prove in the same manner) a counterpart of Theorem 3 for the biased card shuffling: as for the worst-initial condition case, the mixing time simply corresponds to the maximal mixing time of all the associated ASEP projections.

2.4. Some connections with the literature. Benjamini et al. [4] showed that the mixing time of the biased card shuffling is at most of order $N$ ( or rather $N^{2}$ in the discrete time setup considered therein, see also [15]). As a lower bound matching up to a constant $\left(\frac{(1-\alpha) N}{p-q}\right)$ can be obtained by bounding the travel time for the leftmost particle to come to equilibrium (this argument is given in details in [25, Section 5] in the case of small bias), this established pre-cutoff.

As shown in [25] and in the present work, the mixing time for the ASEP and the biased card shuffling is proportional to $(p-q)^{-1} N$ whenever $p>1 / 2$ is fixed. On the other hand when $p=1 / 2$, we know since Wilson [32], that the mixing time is order $N^{2} \log N$. A natural question which was answered by Levin and Peres in [25] is: if the asymmetry scales down to zero, how is the expression for the mixing time modified? The answer, which is given in [25], is that the expression for the mixing time depends on the amount of asymmetry distinguishing between three cases: $(p-q) \geq N^{-1} \log N,(p-q) \in$ $\left[N^{-1}, N^{-1} \log N\right]$ and $(p-q) \leq N^{-1}$. For each of them, an expression for the mixing time is given and pre-cutoff is proved. 
As cutoff holds in both the symmetric and the fully asymmetric cases, it is tempting to conjecture that it should hold for all regimes in between. This problem is the object of a work in preparation [19].

Let us conclude by mentioning the references $[6,16]$ where other types of biased adjacent transpositions are considered: therein, the asymmetry is not fixed but depends on the values of $\sigma(i)$ and $\sigma(i+1)$. For some families of processes of this type, rapid mixing (that is, mixing in polynomial time) is shown. It seems that the question of cutoff is largely open for this type chain.

2.5. Organization of the paper. In Section 3, we introduce alternative descriptions of the ASEP and biased card shuffling in terms of height functions, and we present some monotonicity properties for the dynamics. This is classical in the study of particle systems and ubiquitous in the literature (see [30] for an early reference). Then we present a proof of the identification of the spectral gap using the discrete Hopf-Cole transform. Finally we also treat in that section the particular case of the TASEP (ASEP with $p=1$ ).

In Section 4, we present results (proved in subsequent sections) for the limiting behavior of the particle density (the hydrodynamic limit) and the positions of the leftmost particle and rightmost empty site. Using these results and the materials on the spectral gap, we prove all our main theorems.

In Section 5 we prove the hydrodynamic result by extending the arguments developed in [18] to the constant biased case. Finally, in Section 6 we prove the results concerning convergence of the positions of the leftmost particle and the rightmost empty site by combining hydrodynamic limit estimates with coupling with a stationary variant of ASEP with a finite number of particles on the infinite line.

Sections 4, 5 and 6 are mostly independent and each of them can be read separately.

\section{Technical preliminaries.}

3.1. Notation. In some proofs, it is easier to deal with the following alternative notion of distance to equilibrium

$$
\bar{d}(t):=\max _{\xi, \xi^{\prime} \in \Omega}\left\|P_{t}^{\xi}-P_{t}^{\xi^{\prime}}\right\|_{T V}
$$

This does not raise any issue since the triangle inequality ensures that

$$
d(t) \leq \bar{d}(t) \leq 2 d(t)
$$

We use the same superscript as for $d(t)$ when using the notation $\bar{d}(t)$ for one of the Markov chain introduced above. 


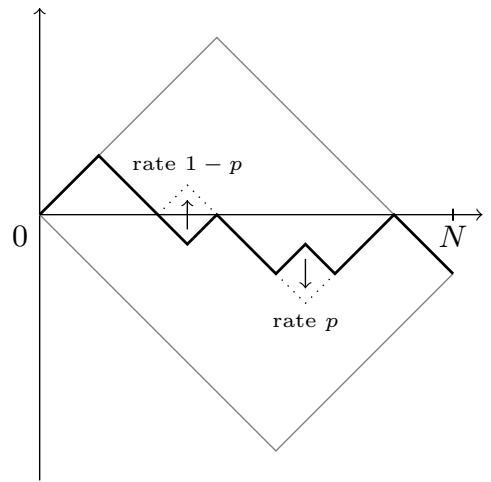

FIG 1. An example of height function with $k=6$ particles over $N=14$ sites. The interface lives within the grey rectangle.

3.2. Height-function, monotone coupling. A classical and convenient equivalent description of the particle system is given by the height function obtained through the mapping $\eta \mapsto h(\eta)$ defined by

$$
\forall x \in \llbracket 0, N \rrbracket, \quad h(\eta)(x):=\sum_{y=1}^{x}(2 \eta(y)-1) .
$$

We let $\Omega_{N, k}:=h\left(\Omega_{N, k}^{0}\right)$ be the set of all such discrete height functions (which happen to be discrete bridges from $(0,0)$ to $(N, 2 k-N))$. We denote by $\left(h^{\xi}(t, \cdot)\right)_{t \geqslant 0}$ the dynamics on the height function: as $\eta \mapsto h(\eta)$ is injective, this contains the same information as the original dynamics and in particular is Markovian.

For the record, let us write the generator of the height-function dynamics. For $f: \Omega_{N, k} \rightarrow \mathbb{R}$,

$$
\mathcal{L}_{N, k} f(\xi):=\sum_{x=1}^{N-1} p\left[f\left(\xi_{x}\right)-f(\xi)\right]+(1-p)\left[f\left(\xi^{x}\right)-f(\xi)\right] .
$$

where the configurations $\xi_{x}$ and $\xi^{x}$ are respectively defined by

$$
\left\{\begin{array}{l}
\xi^{x}(y)=\xi_{x}(y)=\xi(y) \quad \text { for } y \in \llbracket 0, N \rrbracket \backslash\{x\} \\
\xi_{x}(x)=\max (\xi(x+1), \xi(x-1))-1 \\
\xi^{x}(x)=\min (\xi(x+1), \xi(x-1))+1
\end{array}\right.
$$

In words, this simply means that local maxima (resp. minima) flip into local minima (resp. maxima) at rate $p$ (resp. $q$ ), see Figure 3.2. 
Similarly as in (5), we define a function $h_{k}$ that maps $\mathcal{S}_{N}$ to $\Omega_{N, k}$, by setting

$$
h_{k}(\sigma)(x):=\sum_{i=1}^{x}\left(2 \mathbf{1}_{\{\sigma(i) \geq N-k+1\}}-1\right) .
$$

Note that the knowledge of all the height-functions $h_{k}(\sigma), k \in \llbracket 1, N-1 \rrbracket$ is sufficient to recover $\sigma$ completely:

$$
\sigma(x)=N-k+1 \Leftrightarrow\left\{\begin{array}{l}
h_{k}(\sigma)(x)-h_{k}(\sigma)(x-1)=1, \quad \text { and } \\
h_{k-1}(\sigma)(x)-h_{k-1}(\sigma)(x-1)=-1
\end{array}\right.
$$

Hence $\sigma \mapsto\left(h_{k}(\sigma)\right)_{k=1}^{N-1}$ is injective.

The representation offers a very convenient framework to introduce an order on the state-space. The relation " $\geq$ " is defined on $\Omega_{N, k} \times \Omega_{N, k}$, as follows

$$
\left\{\xi_{1} \geq \xi_{2}\right\} \Leftrightarrow\left\{\forall x \in \llbracket 0, N \rrbracket, \xi_{1}(x) \geq \xi_{2}(x)\right\} .
$$

The maximal element, which we denote by $\wedge$, corresponds to the configuration where all particles are on the left, and the minimal element $\vee$ corresponds to that where all particles are on the right. Even though this is not apparent in the notation, these two elements depend on $k$ : we believe this will never raise any confusion in the sequel as the value $k$ will always be clear from the context.

These orders on the spaces $\Omega_{N, k}$ induce an order on the group of permutations $\mathcal{S}_{N}$ also denoted by " $\geq$ ", via the following relation

$$
\left\{\sigma_{1} \geq \sigma_{2}\right\} \Leftrightarrow\left\{\forall k \in \llbracket 1, N-1 \rrbracket, h_{k}\left(\sigma_{1}\right) \geq h_{k}\left(\sigma_{2}\right)\right\}
$$

The minimal element is the identity id and the maximal one is the permutation $\sigma_{\max }$ defined in (4).

These orders are natural to consider since they are in some sense perserved by the dynamics. It is indeed a classical result that we can construct a grand coupling that preserves the order in the following sense.

Proposition 5. There exists a coupling of the processes $\left(\sigma_{t}^{\xi}\right)_{t \geq 0}$ starting from $\xi \in \mathcal{S}_{N}$ which satisfies

$$
\left\{\xi \geq \xi^{\prime}\right\} \Rightarrow\left\{\forall t \geq 0, \sigma_{t}^{\xi} \geq \sigma_{t}^{\xi^{\prime}}\right\}
$$


For any $k \in \llbracket 1, N-1 \rrbracket$ there exists a coupling of the processes $\left(h^{\xi}(t, \cdot)\right)_{t \geq 0}$ starting from $\xi \in \Omega_{N, k}$ such that

$$
\left\{\xi \geq \xi^{\prime}\right\} \Rightarrow\left\{\forall t \geq 0, h^{\xi}(t, \cdot) \geq h^{\xi^{\prime}}(t, \cdot)\right\} .
$$

We include a proof of this result in Appendix A for completeness. Note that only the coupling for the card shuffling needs to be constructed since its projection (5) yields an order preserving coupling on $\Omega_{N, k}$. Dynamics with such order preserving property are usually called attractive. In the rest of the paper, unless it is specified otherwise, we always work with such a grand coupling and use $\mathbb{P}$ to denote the associated probability distribution.

3.3. Identification of the spectral gap. A tool which provides an intuition for identifying the spectral gap as well as the eigenfunctions of the generator $\mathcal{L}_{N, k}$ is the celebrated discrete Hopf-Cole transform, which was originally introduced by Gärtner [14] to derive the hydrodynamic limit of the process in a weakly asymmetric regime (when $p-1 / 2$ scales like $1 / N$ ). Let us recall that the continuous Hopf-Cole transform $u \mapsto V(u)$ defined by $V(u)(t, x):=$ $e^{c u(t, x)}$ allows to map the non-linear parabolic PDE

$$
\partial_{t} u=\frac{1}{2} \partial_{x}^{2} u-\frac{c}{2}\left[1-\left(\partial_{x} u\right)^{2}\right]
$$

onto the linear parabolic PDE

$$
\partial_{t} V=\frac{1}{2} \partial_{x}^{2} V-\frac{c^{2}}{2} V
$$

Note that usually, the transformation which is considered is rather $V(u)(t, x):=$ $e^{c\left[u(t, x)+\frac{c}{2} t\right]}$, but in our case we prefer not to have any time dependence in the expression.

Here, in analogy if one sets $u^{\xi}(t, x):=\mathbb{E}\left[h^{\xi}(t, x)\right]$, then it is not difficult to check from the expression (13) of the generator $\mathcal{L}_{N, k}$ that for all $x \in \llbracket 1, N-1 \rrbracket$ we have

$$
\partial_{t} u^{\xi}(t, x)=\frac{1}{2} \Delta u^{\xi}(t, x)-(p-q) \mathbb{E}\left[\mathbf{1}_{\{h \xi(t, x+1)=h \xi(t, x-1)\}}\right],
$$

where the discrete Laplace operator is defined by

$$
\Delta f(x)=f(x+1)-2 f(x)+f(x-1), \quad x \in \llbracket 1, N-1 \rrbracket .
$$

The second term is the discrete analogue of $\left[1-\left(\partial_{x} u\right)^{2}\right]\left(\partial_{x} u\right.$ being replaced by the mean slope on the interval $[x-1, x+1])$. This equation is non-linear in $u$. 
In order to obtain a linear equation, we perform a discrete Hopf-Cole transform. Due to discretization effects, $u \mapsto e^{2(p-q) u}$ is not the right transformation to consider. Additionally, we have to take care of the boundary conditions. We set

$$
\zeta_{x}(\xi)=\lambda^{\frac{1}{2} \xi(x)} \text { and } \widetilde{V}(t, x)=\mathbb{E}\left[\zeta_{x}\left(h^{\xi}(t, \cdot)\right)\right] .
$$

A computation yields that for all $x \in \llbracket 1, N-1 \rrbracket$,

$$
\mathcal{L}_{N, k}\left(\zeta_{x}\right)(\xi)=\sqrt{p q} \Delta\left(\lambda^{\frac{1}{2} \xi(x)}\right)-\varrho \lambda^{\frac{1}{2} \xi(x)},
$$

where

$$
\varrho:=(\sqrt{p}-\sqrt{q})^{2} .
$$

As $\sqrt{p q} \Delta-\varrho$ is a linear operator on $\mathbb{R}^{\mathbb{Z}}$, it commutes with expectation. This immediately implies that for $x \in \llbracket 1, N-1 \rrbracket$ and $t \geq 0$ we have,

$$
\left\{\begin{array}{l}
\partial_{t} \widetilde{V}(t, x)=(\sqrt{p q} \Delta-\varrho) \widetilde{V}(t, x), \quad x \in \llbracket 1, N-1 \rrbracket, \\
\widetilde{V}(t, 0)=1, \quad \widetilde{V}(t, N)=\lambda^{\frac{2 k-N}{2}}
\end{array}\right.
$$

Finally, to deal with the boundary conditions, we let $a_{N, k}(x)$ be the solution of the following system

$$
\left\{\begin{array}{l}
(\sqrt{p q} \Delta-\varrho) a(x)=0, \quad x \in \llbracket 1, N-1 \rrbracket, \\
a(0)=1, \quad a(N)=\lambda^{\frac{2 k-N}{2}} .
\end{array}\right.
$$

We can check that $V(t, x)=\tilde{V}(t, x)-a_{N, k}(x)$ satisfies for all $t \geq 0$ and $x \in \llbracket 0, N \rrbracket$,

$$
\left\{\begin{array}{l}
\partial_{t} V(t, x)=(\sqrt{p q} \Delta-\varrho) V(t, x), \quad x \in \llbracket 1, N-1 \rrbracket . \\
V(t, 0)=V(t, N)=0
\end{array}\right.
$$

This equation allows to identify some eigenfunctions of $\mathcal{L}_{N, k}$ by considering the decomposition of $V$ on a basis of eigenfunctions of $\sqrt{p q} \Delta-\varrho$. If one sets for $j=1, \ldots, N-1$

$$
f_{N, k}^{(j)}(\xi):=\sum_{x=1}^{N-1} \sin \left(\frac{x j \pi}{N}\right)\left(\lambda^{\frac{1}{2} \xi(x)}-a_{N, k}(x)\right),
$$

then the projection of the equation at time zero on the $j$-th Fourier mode yields

$$
\begin{aligned}
& \mathcal{L}_{N, k} f_{N, k}^{(j)}(\xi)=\sqrt{p q} \sum_{x=1}^{N-1} \sin \left(\frac{x j \pi}{N}\right) \Delta\left(\lambda^{\frac{1}{2} \xi}-a_{N, k}\right)(x)-\varrho f_{N, k}^{(j)}(\xi) \\
& \text { imsart-aop ver. } 2014 / 10 / 16 \text { file: AOP1290.tex date: May } 9,2019
\end{aligned}
$$


Using discrete integration by parts twice (which do not yield boundary terms since both functions vanish at 0 and $N)$, and using the fact that $\sin \left(\frac{\cdot j \pi}{N}\right)$ is an eigenfunction for $\Delta$, we obtain that

$$
\sum_{x=1}^{N-1} \Delta\left(\sin \left(\frac{\cdot j \pi}{N}\right)\right)(x)\left(\lambda^{\frac{1}{2} \xi(x)}-a_{N, k}(x)\right)=2\left(\cos \left(\frac{j \pi}{N}\right)-1\right) f_{N, k}^{(j)}(\xi)
$$

and thus

$$
\mathcal{L}_{N, k} f_{N, k}^{(j)}=-\left(\varrho+\gamma_{N}^{(j)}\right) f_{N, k}^{(j)}
$$

where

$$
\gamma_{N}^{(j)}=2 \sqrt{p q}\left(1-\cos \left(\frac{j \pi}{N}\right)\right)=4 \sqrt{p q}\left[\sin \left(\frac{j \pi}{2 N}\right)\right]^{2} .
$$

Of course, except in the special cases $k=1$ or $k=N-1$, this is far from providing a complete basis of eigenfunctions (there are a total of $\left(\begin{array}{l}N \\ k\end{array}\right)$ of them), but this will turn out to be sufficient to identify the spectral gap.

First, and this is the most obvious part, considering the case $j=1$ (which minimizes the quantity $\left.\varrho+\gamma_{N}^{(j)}\right)$ and setting $f_{N, k}:=f_{N, k}^{(1)}$ and $\gamma_{N}:=\gamma_{N}^{(1)}$, we obtain an upper bound on the spectral gap. This is valid for the ASEP, but also for the biased card shuffling as $f_{N, k} \circ h_{k}$ (recall (14)) is an eigenfunction for $\mathcal{L}_{N}$. Thus we have

$$
\operatorname{gap}_{N, k} \leq \varrho+\gamma_{N} \text { and } \operatorname{gap}_{N} \leq \varrho+\gamma_{N}
$$

To prove that this eigenvalue really corresponds to the spectral gap an important observation is that $f_{N, k}$ is an increasing function on $\Omega_{N, k}$ in the following sense

$$
\forall \xi, \xi^{\prime} \in \Omega_{N, k},\left\{\xi \leq \xi^{\prime}\right\} \Rightarrow\left\{f_{N, k}(\xi) \leq f_{N, k}\left(\xi^{\prime}\right)\right\}
$$

In [10, Section 2.7] it is shown that for a reversible attractive dynamics (see Proposition 5) with a maximal and a minimal element, the eigenfunction corresponding to the spectral gap is increasing. As it is quite difficult for two increasing functions to be orthogonal this indicates that $\varrho+\gamma_{N}$ has to be the spectral gap. We prove it in the next section by making use of the monotone coupling. This is in fact a classical computation for attractive systems (see e.g. [32, Section 3.1]) but we shall include it in full for the sake of completeness. 
3.4. Squeezing with monotone coupling. Let us start with the case of ASEP with $k$ particles. Recall that $\mathbb{P}$ is an order preserving coupling, and that $\vee$ and $\wedge$ denote the minimal and maximal configurations respectively. Order-preserving implies in particular that once the dynamics starting from the two extremal height functions merge, the value of $h^{\xi}(t, \cdot)$ is the same for all $\xi \in \Omega_{N, k}$

$$
\left\{h^{\vee}(t, \cdot)=h^{\wedge}(t, \cdot)\right\} \Rightarrow\left\{\forall \xi \neq \xi^{\prime}, h^{\xi}(t, \cdot)=h^{\xi^{\prime}}(t, \cdot)\right\} .
$$

Using this, we consider $\xi, \xi^{\prime} \in \Omega_{N, k}$ which maximizes the total variation distance at time $t$ (recall (12)) and argue as follows:

$$
\bar{d}_{N, k}(t)=\left\|P_{t}^{\xi}-P_{t}^{\xi^{\prime}}\right\|_{T V} \leq \mathbb{P}\left(h^{\xi}(t, \cdot) \neq h^{\xi^{\prime}}(t, \cdot)\right) \leq \mathbb{P}\left(h_{t}^{\vee} \neq h_{t}^{\wedge}\right) .
$$

Using the monotonicity of $f_{N, k}$, and the Markov inequality, we obtain that the quantity above is smaller than

$$
\mathbb{P}\left(f_{N, k}\left(h_{t}^{\wedge}\right) \geq f_{N, k}\left(h_{t}^{\vee}\right)+\delta_{\min }\left(f_{N, k}\right)\right) \leq \frac{\mathbb{E}\left[f_{N, k}\left(h_{t}^{\wedge}\right)-f_{N, k}\left(h_{t}^{\vee}\right)\right]}{\delta_{\min }\left(f_{N, k}\right)}
$$

where

$$
\delta_{\min }\left(f_{N, k}\right)=\min _{\substack{\xi, \xi^{\prime} \in \Omega_{N, k} \\ \xi \geq \xi^{\prime} \text { and } \xi \neq \xi^{\prime}}}\left(f_{N, k}(\xi)-f_{N, k}\left(\xi^{\prime}\right)\right) .
$$

By (18), we deduce that

$$
d_{N, k}(t) \leq \bar{d}_{N, k}(t) \leq \frac{\mathbb{E}\left[f_{N, k}\left(h_{t}^{\wedge}\right)-f\left(h_{t}^{\vee}\right)\right]}{\delta_{\min }\left(f_{N, k}\right)}=\frac{f_{N, k}(\wedge)-f_{N, k}(\vee)}{\delta_{\min }\left(f_{N, k}\right)} e^{-\left(\gamma_{N}+\varrho\right) t}
$$

In view of $(2)$ this implies that $\operatorname{gap}_{N, k} \geq\left(\gamma_{N}+\varrho\right)$ so that we conclude that (8) holds.

Let us mention here that for all $k, N$,

$$
\delta_{\min }\left(f_{N, k}\right)=\min _{x \in \llbracket 1, N-1 \rrbracket} \sin \left(\frac{x \pi}{N}\right)(\lambda-1) \lambda^{\vee(x)} \geq \frac{(\lambda-1)}{N} \lambda^{\frac{k-N}{2}} .
$$

Regarding (3), we observe as above that for all $\xi, \xi^{\prime} \in \mathcal{S}_{N}$ which maximize the total variation distance at time $t$, we have

$$
\bar{d}_{N}(t)=\left\|Q_{t}^{\xi}-Q_{t}^{\xi^{\prime}}\right\|_{T V} \leq \mathbb{P}\left(\sigma_{t}^{\xi} \neq \sigma_{t}^{\xi^{\prime}}\right)
$$


By injectivity of the height-function (recall (15)) we have

$$
\begin{aligned}
\mathbb{P}\left(\sigma_{t}^{\xi} \neq \sigma_{t}^{\xi^{\prime}}\right) & =\mathbb{P}\left(\exists k \in \llbracket 1, N-1 \rrbracket, h_{k}\left(\sigma_{t}^{\xi}\right) \neq h_{k}\left(\sigma_{t}^{\xi^{\prime}}\right)\right) \\
& \leq \sum_{k=1}^{N} \mathbb{P}\left(h_{k}\left(\sigma_{t}^{\xi}\right) \neq h_{k}\left(\sigma_{t}^{\xi^{\prime}}\right)\right) .
\end{aligned}
$$

Then repeating (19) and (22), it follows that each term in the last sum is smaller than $\left(f_{N, k}(\wedge)-f_{N, k}(\vee)\right) e^{-\left(\gamma_{N}+\varrho\right) t}$, which allows to conclude that (3) holds.

To conclude let us remark that the above method provides us a quantitative upper-bound on the mixing time, but that it does not allow to identify the right constant. More precisely (details are left to the reader), we have

$$
\bar{d}_{N, k}(t) \leq C_{\lambda} N \lambda^{N / 2} e^{-\left(\gamma_{N}+\varrho\right) t}, \quad \bar{d}(t) \leq C_{\lambda} N^{2} \lambda^{N / 2} e^{-\left(\gamma_{N}+\varrho\right) t},
$$

for some constant $C_{\lambda}>0$ depending on $\lambda$. This gives an upper bound (which perhaps surprisingly does not depend on $k$ ) of order $\frac{(\log \lambda)}{2 \varrho} N$ for both mixing times. While this is the right order of magnitude for the mixing time, the constant in front is not optimal. However, it can be remarked that for vanishing asymmetry, it gets asymptotically close to the right one for the case of biased shuffle or when $k=N / 2$.

3.5. The special case of $p=1$ : TASEP. Let us make some comments here about the case $p=1$ for which Theorem 2 is a simple consequence of the work of [30]. In that case, the system is mixed when $h^{\xi}(t, \cdot)$ hits the lowest configuration $\vee$ (in particular by monotonicity $\wedge$ is the worst configuration to start with).

Another remark is that $h^{\wedge}(t, \cdot)$ can be coupled with a TASEP dynamics on the infinite line with height function $h^{\wedge, \infty}(t, \cdot)$ starting from

$$
\wedge_{\infty}(x):=x \mathbf{1}_{\{x \leq k\}}+(2 k-x) \mathbf{1}_{\{x>k\}},
$$

in such a way that for all $t$ and $x$

$$
h^{\wedge}(t, \cdot):=\max \left(\vee(x), h^{\wedge, \infty}(t, \cdot)\right) .
$$

Hence we have when $p=1$

$$
d^{N, k}(t)=\mathbb{P}\left[h^{\wedge}(t, \cdot) \neq \vee\right]=\mathbb{P}\left[h^{\wedge, \infty}(t, N-k)>-(N-k)\right] .
$$

Then it follows from [30, Theorem 1] that when $k / N \rightarrow \alpha$, the limit is one if one chooses $t=N\left[(\sqrt{\alpha}+\sqrt{1-\alpha})^{2}-\varepsilon\right]$ and the limit is zero if $t=N\left[(\sqrt{\alpha}+\sqrt{1-\alpha})^{2}+\varepsilon\right]$, thus yielding Theorem 2 . 
Since the work of Rost, much more detailed results have in fact been obtained about the scaling for $h^{\wedge, \infty}(t, \cdot)$ and its fluctuations are known [13, Equation (3.7)] to be described by the Airy ${ }_{2}$ process. This information allows to deduce that when $k=N / 2, d^{N, N / 2}(t)$ drops from one to zero in a time window of order $N^{1 / 3}$ and even to identify the limit of $d^{N, N / 2}\left(2 N+N^{1 / 3} u\right)$, as a function of $u$ that can be expressed in terms of the distribution of the Airy $_{2}$ process.

An important thing to keep in mind is that a coupling such as (26) does not exist when $p<1$, the reason being that the boundary condition have the effect of pushing $h(t, \cdot)$ in the upward direction. This is what makes the analysis of ASEP more difficult, and the main reason why the question of cutoff has been open for more than a decade. While we do believe that the statement about the $N^{1 / 3}$ cutoff window and profile should remain valid when $p \in(0,1)$, and also for every $\alpha \in(0,1)$, they remain at this stage, challenging conjectures.

4. Getting the mixing times from scaling limits. In the present section, we show how to reduce the proof of the mixing time for both processes to a scaling limit statement about the positions of the leftmost particle and the rightmost empty site (Proposition 9). The underlying idea is that the contraction inequality derived from the eigenfunction $f_{N, k}$, while not providing a sharp estimate on the mixing time, allows to prove that once the system is macroscopically close to equilibrium, it mixes rapidly.

4.1. The hydrodynamic profile. Let $\xi_{N}$ be a sequence of elements of $\cup_{k} \Omega_{N, k}^{0}$ and let us define the associated sequence of empirical densities

$$
\rho_{t}^{N}(d y)=\frac{1}{N} \sum_{x=1}^{N} \eta^{\xi_{N}}\left(\frac{N t}{p-q}, x\right) \delta_{x / N}(d y)
$$

Notice that $\rho_{t}^{N}$ belongs to the convex set $\mathcal{M}$ of measures on $[0,1]$ with totalmass at most 1 . We endow this set with the topology of weak convergence, see $(11)$.

We assume that $\rho_{0}^{N}$ converges weakly to some limiting density $\rho_{0}$ : this is a harmless assumption since the sequence $\rho_{0}^{N}$ is tight. As $\left\langle\rho_{0}^{N}, \varphi\right\rangle \leqslant \frac{1}{N} \sum_{x=1}^{N} \varphi(x / N)$ for any $\varphi \in \mathcal{C}\left([0,1], \mathbb{R}_{+}\right)$, it is simple to check that $\rho_{0}$ necessarily belongs to the dual of $L^{1}$, namely to $L^{\infty}$, and satisfies $\rho_{0}(x) \in[0,1]$ for almost every $x \in[0,1]$. We also let $h^{\xi_{N}}(t, x)$ be the associated height function, and we define $u^{N}(t, x)=\frac{1}{N} h\left(\frac{N}{p-q} t, x N\right)$ for all $t \geqslant 0$ and $x \in[0,1]$. 
TheOREM 4. The sequence $\rho^{N}$ converges in distribution in the Skorohod space $\mathbb{D}\left(\mathbb{R}_{+}, \mathcal{M}\right)$ towards the unique entropy solution of the inviscid Burgers equation with zero-flux boundary conditions:

$$
\left\{\begin{array}{lr}
\partial_{t} \rho=-\partial_{x}(\rho(1-\rho)), & t>0, x \in(0,1) \\
\rho(t, x)(1-\rho(t, x))=0, & t>0, x \in\{0,1\} \\
\rho(0, \cdot)=\rho_{0}(\cdot) &
\end{array}\right.
$$

Furthermore, the sequence $u^{N}$ converges in $\mathbb{D}\left(\mathbb{R}_{+}, \mathcal{C}([0,1])\right)$ towards the integrated solution $u(t, x)=\int_{0}^{x}(2 \rho(t, y)-1) \mathrm{d} y$.

The Skorohod spaces denote the spaces of cadlag functions and are endowed with the Skorohod topology, see Billingsley [7].

REMARK 6. Note that intuitively $u$ should be the solution of the following equation

$$
\left\{\begin{array}{l}
\partial_{t} u=-\frac{1}{2}\left(1-\left(\partial_{x} u\right)^{2}\right), \quad t>0, x \in(0,1) \\
u(0, \cdot)=0 \quad \text { and } \quad u(1, \cdot)=2 \alpha-1, \quad t>0, x \in\{0,1\} \\
u(0, \cdot)=\int_{0}^{\cdot}(2 \rho(t, y)-1) \mathrm{d} y
\end{array}\right.
$$

However, the precise connection between (27) and (28) has not been established in the literature. So we stick to the problem (27) formulated in terms of particle density, as it is sufficient to our purpose.

REMARK 7. All the solutions of (27) obtained in Theorem 4 stabilize in finite time to an equilibrium profile, given by $\mathbf{1}_{[1-\alpha, 1]}$ where $\alpha=\int_{[0,1]} \rho_{0}(x) \mathrm{d} x$. Indeed, the explicit solution starting from $\wedge$ stabilizes in finite time and stays above any other solution, by monotonicity of the particle system. We define thus

$$
t_{\rho_{0}}:=\inf \left\{t>0 \mid \rho(t, \cdot)=\mathbf{1}_{[1-\alpha, 1]}\right\} .
$$

This is the quantity involved in the expression of the mixing time in Theorem 3. Note that while in the extremal case described in Theorem 2, the mixing time coincides with $N(p-q)^{-1} t_{\rho_{0}}$ for $\rho_{0}:=\mathbf{1}_{[0, \alpha]}$, this is not always the case when starting from an arbitrary condition as the position of the leftmost particle and rightmost empty site can, in some cases, take a longer time to reach equilibrium than $\rho$.

The precise definition of the entropy solutions of (27) as well as the proof of the convergence is postponed to Section 5 . 
Let us describe the scaling limit of the height function starting from the maximal element $\wedge$. This object is relevant only when the density of particle $\alpha$ is strictly positive: at the end of the present subsection, we introduce the relevant quantities when $\alpha=0$. For $\alpha \in(0,1 / 2]$, we define $\vee_{\alpha}:[0,1] \rightarrow \mathbb{R}$, $\wedge_{\alpha}:[0,1] \rightarrow \mathbb{R}$ which correspond to the extremal macroscopic states,

$$
\vee_{\alpha}(x):=\max (-x, x-2(1-\alpha)), \quad \wedge_{\alpha}(x):=\min (x, 2 \alpha-x),
$$

and let $g_{\alpha}: \mathbb{R}_{+} \times[0,1] \rightarrow \mathbb{R}$ be defined as follows

$$
\begin{aligned}
& g_{\alpha}^{0}(t, x):= \begin{cases}\alpha-\frac{t}{2}-\frac{(x-\alpha)^{2}}{2 t}, & \text { if }|x-\alpha| \leq t, \\
\wedge^{\alpha}(x), & \text { if }|x-\alpha| \geq t,\end{cases} \\
& g_{\alpha}(t, x):=\max \left(\vee_{\alpha}(x), g_{\alpha}^{0}(x, t)\right) .
\end{aligned}
$$

As a consequence of Theorem 4, we obtain the following statement.

Corollary 8. Let $p \in(1 / 2,1]$ and $\alpha \in(0,1 / 2]$. For any $\varepsilon>0$ and any $T>0$, we have

$$
\lim _{N \rightarrow \infty} \mathbb{P}\left[\sup _{t \in[0, T]} \sup _{x \in[0,1]}\left|\frac{1}{N} h^{\wedge}\left(\frac{N t}{p-q}, N x\right)-g_{\alpha}(t, x)\right| \leq \varepsilon\right]=0 .
$$

Let us introduce $\ell_{\alpha}(t), r_{\alpha}(t) \in[0,1]$, which for $t \leq(\sqrt{\alpha}+\sqrt{1-\alpha})^{2}$ are the extremities of the interval on which $g_{\alpha}(t, \cdot)$ and $g_{\alpha}^{0}(t, x)$ coincide):

$$
\ell_{\alpha}(t)= \begin{cases}0 & \text { if } t \leq \alpha \\ (\sqrt{t}-\sqrt{\alpha})^{2} & \text { if } t \in\left(\alpha,(\sqrt{\alpha}+\sqrt{1-\alpha})^{2}\right) \\ 1-\alpha & \text { if } t \geq(\sqrt{\alpha}+\sqrt{1-\alpha})^{2}\end{cases}
$$

and

$$
r_{\alpha}(t)= \begin{cases}1 & \text { if } t \leq 1-\alpha \\ 1-(\sqrt{t}-\sqrt{1-\alpha})^{2} & \text { if } t \in\left(1-\alpha,(\sqrt{\alpha}+\sqrt{1-\alpha})^{2}\right) \\ 1-\alpha & \text { if } t \geq(\sqrt{\alpha}+\sqrt{1-\alpha})^{2} .\end{cases}
$$

When $\alpha=0$, the hydrodynamic limit does not evolve since the system is macroscopically at equilibrium at time 0 even though it is far from the microscopical equilibrium. We introduce:

$$
\begin{aligned}
& \ell_{0}(t)=t \wedge 1 \quad \text { and } \quad r_{0}(t)=1, \quad t \geq 0 \\
& \text { imsart-aop ver. 2014/10/16 file: AOP1290.tex date: May 9, } 2019
\end{aligned}
$$


4.2. Scaling limit for rightmost particle and leftmost empty site. We introduce now the key statements that will allow us to get sharp mixing time estimates: given $\xi \in \Omega_{N, k}$, we let $\ell_{N, k}(\xi)$ and $r_{N, k}(\xi)$ denote the position of the leftmost particle and the rightmost empty site respectively (which almost corresponds to the quantities introduced in (9) for $\xi \in \Omega_{N, k}^{0}$ ). In terms of height function this translates into

$$
\begin{aligned}
& \ell_{N, k}(\xi)=\max \{x \in \llbracket 0, N \rrbracket: \xi(x)=-x\}, \\
& r_{N, k}(\xi)=\min \{x \in \llbracket 0, N \rrbracket: \xi(x)=x-2(N-k)\} .
\end{aligned}
$$

We also set

$$
L_{N, k}(t):=\ell_{N, k}\left(h_{t}^{\wedge}\right) \quad \text { and } \quad R_{N, k}(t):=r_{N, k}\left(h_{t}^{\wedge}\right) .
$$

Our result is the following.

Proposition 9. Let $p \in(1 / 2,1]$ and $\alpha \in[0,1 / 2]$. For any $t \geqslant 0$ we have the following convergences in probability

$$
\lim _{\substack{N \rightarrow \infty \\ k / N \rightarrow \alpha}} N^{-1} L_{N, k}\left(\frac{N t}{p-q}\right)=\ell_{\alpha}(t) \text { and } \lim _{\substack{N \rightarrow \infty \\ k / N \rightarrow \alpha}} N^{-1} R_{N, k}\left(\frac{N t}{p-q}\right)=r_{\alpha}(t) .
$$

Let us stress that in the case where $\alpha \in(0,1 / 2]$, Corollary 8 does not directly imply Proposition 9. It rather states that $\ell_{\alpha}(t)$ is the smallest point where the particle density is non-zero and $r_{\alpha}(t)$ is the largest point where it is not equal to 1 so that, provided the limit exits in probability, one deduces the upper bound for $L_{N, k}$ and the lower bound for $R_{N, k}$ :

$$
\lim _{\substack{N \rightarrow \infty \\ k / N \rightarrow \alpha}} N^{-1} L_{N, k}\left(\frac{N t}{p-q}\right) \leqslant \ell_{\alpha}(t) \text { and } \lim _{\substack{N \rightarrow \infty \\ k / N \rightarrow \alpha}} N^{-1} R_{N, k}\left(\frac{N t}{p-q}\right) \geqslant r_{\alpha}(t) .
$$

However it does not give the microscopic information that would be necessary to obtain the lower bound for $L_{N, k}$ and the upper bound for $R_{N, k}$. Another important observation is that this microscopic information is not contained in our proof of Theorem 4: the technique we use to derive $g_{\alpha}(t, x)$ as a scaling limit, is to show that the particle density is an entropy solution of the inviscid Burgers equation, and this formulation of the problem does not allow to track the positions of the leftmost particle and the rightmost empty site.

Let us now introduce

$$
\begin{aligned}
A_{N, k}^{\varepsilon}:=\left\{\xi \in \Omega_{N, k}:\left|\ell_{N, k}(\xi)-N+k\right| \leq \varepsilon N \text { and }\left|r_{N, k}(\xi)-N+k\right| \leq \varepsilon N\right\} \\
\text { imsart-aop ver. } 2014 / 10 / 16 \text { file: AOP1290.tex date: May 9, } 2019
\end{aligned}
$$


and

$$
t_{\alpha, N}:=\frac{N}{p-q}(\sqrt{\alpha}+\sqrt{1-\alpha})^{2} .
$$

As a direct consequence of Proposition 9, we get the following result.

Corollary 10. Let $p \in(1 / 2,1]$ and $\alpha \in[0,1 / 2]$. We have for any $\delta \geq 0$ and $\varepsilon>0$

$$
\lim _{\substack{N \rightarrow \infty \\ k / N \rightarrow \alpha}} \mathbb{P}\left(h_{(1+\delta) t_{\alpha, N}}^{\wedge} \in A_{N, k}^{\varepsilon}\right)=1 .
$$

Moreover for any fixed $\delta>0$, for $\varepsilon \leq \varepsilon_{0}(\delta)$ we have

$$
\lim _{\substack{N \rightarrow \infty \\ k / N \rightarrow \alpha}} \mathbb{P}\left(h_{(1-\delta) t_{\alpha, N}}^{\wedge} \in A_{N, k}^{\varepsilon}\right)=0
$$

Finally in order to use these results, we need to check that the final positions of $\ell_{\alpha}, r_{\alpha}$ correspond indeed to equilibrium. This is a known estimate but we include a proof for completeness.

LEMma 11. We have for all values of $N$ and $k$

$$
\pi_{N, k}\left(\left|\ell_{N, k}-r_{N, k}\right| \geq M\right) \leq \frac{\lambda^{3-M}(M+1)}{(\lambda-1)^{2}} .
$$

Proof. As in [25, Proof of Proposition 11], we rely on the observation that if $\ell_{N, k}(\xi)=i-1, r_{N, k}(\xi)=j$ (not both equal to $N-k$ ), then there is a particle at site $i$ while site $j$ is empty, and therefore, the bijection $T_{i, j}$ that interchanges the contents of sites $i$ and $j$ in the particle system, decreases $A(\xi)$ (recall $(7))$ by an amount $j-i$. Thus we have

$$
\pi_{N, k}\left(\ell_{N, k}=i-1, r_{N, k}=j\right) \leq \lambda^{-(j-i)} \sum_{\xi \in \Omega_{N, k}} \pi_{N, k}\left(T_{i, j}(\xi)\right) \leq \lambda^{-(j-i)},
$$

Summing over all possibilities for $i$ and $j$ (at most $A$ possibilities when the gap is equal to $A$ ) this yields

$$
\pi_{N, k}\left(\left|\ell_{N, k}-r_{N, k}\right| \geq M\right) \leq \sum_{A \geq M} A \lambda^{-(A-1)}=\frac{M \lambda^{-(M-1)}}{\left(1-\lambda^{-1}\right)}+\frac{\lambda^{-M}}{\left(1-\lambda^{-1}\right)^{2}}
$$


4.3. The mixing time for ASEP: Proof of Theorem 2. As the case $p=1$ was treated in Section 3.5, we assume here that $p \in(1 / 2,1)$. We have to prove that in the limit when $k / N$ tends to $\alpha$, the mixing time is equivalent to $t_{\alpha, N}$.

The easiest part is to show that the mixing time is at least $t_{\alpha, N}$, or more precisely, that for any $\delta>0$

$$
\lim _{\substack{N \rightarrow \infty \\ k / N \rightarrow \alpha}} d_{N, k}\left((1-\delta) t_{\alpha, N}\right)=1 .
$$

From the definitions of total variation distance and $d_{N, k}$, we have for any $\varepsilon>0$

$$
d_{N, k}\left((1-\delta) t_{\alpha, N}\right) \geq \pi_{N, k}\left(A_{N, k}^{\varepsilon}\right)-P_{(1-\delta) t_{\alpha, N}}^{\wedge}\left(A_{N, k}^{\varepsilon}\right) .
$$

The first probability converges to 1 according to Lemma 11, while the second converges to zero if $\varepsilon$ is sufficiently small according to Corollary 10.

To obtain the other bound on the mixing time, we show that for any $\delta>0$,

$$
\lim _{\substack{N \rightarrow \infty \\ k / N \rightarrow \alpha}} d_{N, k}\left((1+\delta) t_{\alpha, N}\right)=0
$$

Recall (20) and the monotone grand coupling. We have

$$
d^{N, k}\left((1+\delta) t_{\alpha, N}\right) \leq \mathbb{P}\left[h_{(1+\delta) t_{\alpha, N}}^{\wedge} \neq h_{(1+\delta) t_{\alpha, N}}^{\vee}\right]
$$

The right-hand side is smaller than

$$
\mathbb{P}\left[h_{(1+\delta) t_{\alpha, N}}^{\wedge} \neq h_{(1+\delta) t_{\alpha, N}}^{\vee} \mid h_{t_{\alpha, N}}^{\wedge} \in A_{N, k}^{\varepsilon}\right]+\mathbb{P}\left[h_{t_{\alpha, N}}^{\wedge} \notin A_{N, k}^{\varepsilon}\right] .
$$

According to (32), the second term vanishes in the limit. Regarding the first term, using the Markov property and repeating the computation of Section 3.4 , we obtain

$$
\begin{aligned}
& \mathbb{P}\left[h_{(1+\delta) t_{\alpha, N}}^{\wedge} \neq h_{(1+\delta) t_{\alpha, N}}^{\vee} \mid h_{t_{\alpha, N}}^{\wedge}, h_{t_{\alpha, N}}^{\vee}\right] \\
& \leq \mathbb{E}\left[\frac{f_{N, k}\left(h_{(1+\delta) t_{\alpha, N}}^{\wedge}\right)-f_{N, k}\left(h_{(1+\delta) t_{\alpha, N}}^{\vee}\right)}{\delta_{\min }\left(f_{N, k}\right)} \mid h_{t_{\alpha, N}}^{\wedge}, h_{t_{\alpha, N}}^{\vee}\right] \\
& \leq \frac{f_{N, k}\left(h_{t_{\alpha, N}}^{\wedge}\right)-f_{N, k}\left(h_{t_{\alpha, N}}^{\vee}\right)}{\delta_{\min }\left(f_{N, k}\right)} e^{-\delta t_{\alpha, N}\left(\varrho+\gamma_{N}\right)} . \\
& \text { imsart-aop ver. 2014/10/16 file: AOP1290.tex date: May 9, } 2019
\end{aligned}
$$


To conclude we remark that if one defines $\wedge_{N, k}^{\varepsilon}$ to be the maximal element of $A_{N, k}^{\varepsilon}$ (which is well defined since the maximum of two elements of $A_{N, k}^{\varepsilon}$ is in $A_{N, k}^{\varepsilon}$, then for some constant $C=C_{\lambda}>0$, we have on the event $\left\{h_{t_{\alpha, N}}^{\wedge} \in A_{N, k}^{\varepsilon}\right\}$

$$
f_{N, k}\left(h_{t_{\alpha, N}}^{\wedge}\right)-f_{N, k}\left(h_{t_{\alpha, N}}^{\vee}\right) \leq f_{N, k}\left(\wedge_{N, k}^{\varepsilon}\right)-f_{N, k}(\vee) \leq C N \delta_{\min }\left(f_{N, k}\right) \lambda^{\varepsilon N}
$$

where we have used (23). Hence we deduce from (35) that

$$
\mathbb{P}\left[h_{(1+\delta) t_{\alpha, N}}^{\wedge} \neq h_{(1+\delta) t_{\alpha, N}}^{\vee} \mid h_{t_{\alpha, N}}^{\wedge} \in A_{N, k}^{\varepsilon}\right] \leq C N \lambda^{\varepsilon N} e^{-\delta t_{\alpha, N}\left(\varrho+\gamma_{N}\right)} .
$$

If $\varepsilon$ is chosen small compared to $\delta$, this last term tends to zero exponentially fast and we can conclude that (34) holds.

4.4. From ASEP to card-shuffle: Proof of Theorem 1. Let $\sigma_{t}^{\max }$ and $\sigma_{t}^{\min }$ denote the dynamics starting from the maximal $\left(\sigma^{\max }\right.$ from $\left.(4)\right)$ and minimal (the identity) initial conditions. In order to adapt the method used above for the ASEP we need to control the height functions for all levels $k$. We extend the definition (30) to the group of permutations by setting for $\xi \in \mathcal{S}_{N}$

$$
\ell_{N, k}(\xi):=\ell_{N, k}\left(h_{k}(\xi)\right) \quad \text { and } \quad r_{N, k}(\xi):=r_{N, k}\left(h_{k}(\xi)\right)
$$

We also set

$$
\begin{aligned}
B_{N}^{\varepsilon}:=\left\{\xi \in \mathcal{S}_{N}: \forall k \in \llbracket 1, N-1 \rrbracket,\right. \\
\left.\left|\ell_{N, k}(\xi)-N+k\right| \leq \varepsilon N,\left|r_{N, k}(\xi)-N+k\right| \leq \varepsilon N\right\} . \\
=\left\{\xi \in \mathcal{S}_{N}: \forall k \in \llbracket 1, N-1 \rrbracket, h_{k}(\xi) \in A_{N, k}^{\varepsilon}\right\} .
\end{aligned}
$$

We need the following improvement of Corollary 10. We set

$$
t_{N}:=t_{1 / 2, N}=2(p-q)^{-1} N
$$

LEMma 12. We have for any $\varepsilon>0$

$$
\lim _{N \rightarrow \infty} \mathbb{P}\left[\sigma_{t_{N}}^{\max } \in B_{N}^{\varepsilon}\right]=1
$$

Proof. Let $m>0$ be a fixed integer and fix $k_{1}^{N}<\cdots<k_{m}^{N}$ for $N \geq 0$, such that

$$
\forall i \in \llbracket 1, m \rrbracket, \lim _{N \rightarrow \infty} k_{i}^{N} / N=\frac{i}{m+1},
$$


Set

$$
\begin{aligned}
B_{N, m}^{\varepsilon} & :=\left\{\xi \in \mathcal{S}_{N}: \forall i \in \llbracket 1, m \rrbracket,\right. \\
& \left.\left.\left|\ell_{N, k_{i}}(\xi)-N+k_{i}\right| \leq(\varepsilon / 2) N \text { and }\left|r_{N, k_{i}}(\xi)-N+k_{i}\right| \leq(\varepsilon / 2) N\right)\right\} .
\end{aligned}
$$

For a given $\xi \in \mathcal{S}_{N}, \ell_{N, k}(\xi)$ and $r_{N, k}(\xi)$ are non-increasing functions of $k$ (when $k$ increases, only new particles are added). Thus, we have for $m \geq$ $3 \varepsilon^{-1}$ and $N$ large enough $B_{N, m}^{\varepsilon} \subset B_{N}^{\varepsilon}$. Consequently

$$
\begin{aligned}
\mathbb{P}\left[\sigma_{t_{N}}^{\max } \notin B_{N}^{\varepsilon}\right] \leq \mathbb{P}\left[\sigma_{t_{N}}^{\max } \notin B_{N, m}^{\varepsilon}\right] & \leq \sum_{i=1}^{m} \mathbb{P}\left[h_{k}\left(\sigma_{t_{N}}^{\max }\right) \notin A_{N, k_{i}}^{\varepsilon / 2}\right] \\
& =\sum_{i=1}^{m} \mathbb{P}\left[h_{t_{N}}^{\wedge} \notin A_{N, k_{i}}^{\varepsilon / 2}\right] .
\end{aligned}
$$

Using Corollary 10, and the fact that $t_{N}=\max _{\alpha \in[0,1]} t_{N, \alpha}$, we can conclude.

Proof of Theorem 1. Note that we only need to prove an upper-bound since the lower bound is given by the case $\alpha=1 / 2$ of Theorem 2 . We consider separately the case $p=1$ in the next subsection. Recall (24). We have for all $\delta>0$

$$
d_{N}\left((1+\delta) t_{N}\right) \leq \mathbb{P}\left[\sigma_{(1+\delta) t_{N}}^{\max } \neq \sigma_{(1+\delta) t_{N}}^{\min } \mid \sigma_{t_{N}}^{\max } \in B_{N}^{\varepsilon}\right]+\mathbb{P}\left[\sigma_{t_{N}}^{\max } \notin B_{N}^{\varepsilon}\right] .
$$

From Lemma 12, the second term goes to zero. To estimate the first one, we use a conditional version of (25) and (22) and we obtain

$$
\begin{aligned}
& \mathbb{P}\left[\sigma_{(1+\delta) t_{N}}^{\max } \neq \sigma_{(1+\delta) t_{N}}^{\min } \mid \sigma_{t_{N}}^{\max }, \sigma_{t_{N}}^{\min }\right] \\
& \leq \sum_{k=1}^{N} \frac{f_{N, k}\left(h_{k}\left(\sigma_{t_{N}}^{\max }\right)\right)-f_{N, k}\left(h_{k}\left(\sigma_{t_{N}}^{\min }\right)\right)}{\delta_{\min }\left(f_{N, k}\right)} e^{-\delta t_{N}\left(\varrho+\gamma_{N}\right)} .
\end{aligned}
$$

Now if $\sigma_{t_{N}}^{\max }$ is in $B_{N}^{\varepsilon}$ then all its projections are in the respective $A_{N, k}^{\varepsilon}$ and thus (recall that $\wedge_{N, k}^{\varepsilon}$ is the maximal element of $A_{N, k}^{\varepsilon}$ )

$$
f_{N, k}\left(h_{k}\left(\sigma_{t_{N}}^{\max }\right)\right)-f_{N, k}\left(h_{k}\left(\sigma_{t_{N}}^{\min }\right)\right) \leq f_{N, k}\left(\wedge_{N, k}^{\varepsilon}\right)-f_{N, k}(\vee) \leq C N \delta_{\min }\left(f_{N, k}\right) \lambda^{\varepsilon N} .
$$

Thus taking the conditional expectation and applying Lemma 12 we deduce that there exists $C^{\prime}>0$ such that for all $N$ large enough

$$
\begin{aligned}
& \mathbb{P}\left[\sigma_{(1+\delta) t_{N}}^{\max } \neq \sigma_{(1+\delta) t_{N}}^{\min } \mid \sigma_{t_{N}}^{\max } \in B_{N}^{\varepsilon}\right] \leq C^{\prime} N^{2} \lambda^{\varepsilon N} e^{-\delta t_{N}\left(\varrho+\gamma_{N}\right)} . \\
& \text { imsart-aop ver. } 2014 / 10 / 16 \text { file: AOP1290.tex date: May 9, } 2019
\end{aligned}
$$


The right hand side tends to zero exponentially fast provided $\varepsilon$ is chosen sufficiently small compared to $\delta$. Therefore, we have shown that $d_{N}\left((1+\delta) t_{N}\right)$ goes to 0 as $N \rightarrow \infty$, thus concluding the proof of Theorem 1 in the case $p<1$.

4.5. The case $p=1$ for Theorem 1 . Unlike for the TASEP, the mixing for the totally biased card shuffling cannot be obtained directly from [30, Theorem 1]. The reason being that for doing so one would need to know not only the limiting behavior of the hitting time of $\vee_{N, k}$, but also some estimates on the rate of convergence. While this could be achieved by using large deviation results obtained for the TASEP (see e.g. [11]), we prefer in this section to show how the case $p=1$ can be deduced from $p<1$ via approximations. We proceed in two steps.

First, given $\varepsilon$ sufficiently small (independent of $N$ ), we let $\left(\sigma_{t}\right)_{t \geq 0}$ and $\left(\tilde{\sigma}_{t}\right)_{t \geq 0}$ be biased card shuffles with respective asymmetries given by $p=1$, and $\tilde{p}=1-\epsilon / 2$, both with initial condition $\sigma^{\max }$. We denote by $\tilde{\pi}_{N}$ the equilibrium measure associated to $\tilde{\sigma}$. We couple these two processes (using a construction similar to the one displayed in Appendix A) in such a way that for all $t \geq 0$,

$$
\tilde{\sigma}_{t} \geq \sigma_{t}
$$

Notice that for $N$ sufficiently large, Theorem 1 for $\tilde{p}$ yields that $t_{1}:=2 N(1+$ $2 \varepsilon)$ is larger than the $\varepsilon$-mixing time of $\tilde{\sigma}_{t}$. We introduce

$$
\begin{aligned}
\tilde{B}_{N}:=\left\{\xi \in \mathcal{S}_{N}:\right. & : \forall k \in \llbracket 1, N \rrbracket, \\
& \left.\ell_{N, k}(\xi) \geq N-k-\sqrt{N} \text { and } r_{N, k}(\xi) \leq N-k+\sqrt{N}\right\} .
\end{aligned}
$$

As $\tilde{B}_{N}$ is a decreasing event, for $N$ large enough we have from (37) and the mixing estimate for $\tilde{p}$

$$
\mathbb{P}\left[\sigma_{t_{1}} \notin \tilde{B}_{N}\right] \leq \mathbb{P}\left[\tilde{\sigma}_{t_{1}} \notin \tilde{B}_{N}\right] \leq \tilde{\pi}_{N}\left(\mathcal{S}_{N} \backslash \tilde{B}_{N}\right)+\varepsilon \leq 2 \varepsilon .
$$

where the last estimate is obtained using Lemma 11 and a union bound over $k$.

We use then a second coupling for $t \geq t_{1}$ : Let $\left(\hat{\sigma}_{t}\right)_{t \geq t_{1}}$ be a biased shuffling with asymmetry $\hat{p}=1-N^{-2}$ and initial condition $\sigma_{t_{1}}$, and coupled with $\left(\sigma_{t}\right)_{t \geq t_{1}}$ in a way such that at all time $\hat{\sigma}_{t} \geq \sigma_{t}^{\max }$. Also, we let $\hat{\sigma}_{t}^{\min }$ be a shuffling with bias $\hat{p}$ starting from the identity at time $t_{1}$, and couple it in

imsart-aop ver. 2014/10/16 file: AOP1290.tex date: May 9, 2019 
a way such that $\hat{\sigma}_{t} \geq \hat{\sigma}_{t}^{\min }$. We set $t_{2}=t_{1}+\varepsilon N$, and we denote by $\hat{\pi}_{N}$ the equilibrium measure associated to $\hat{\sigma}$. We have

$d_{N}\left(t_{2}\right)=\mathbb{P}\left[\sigma_{t_{2}} \neq \mathrm{id}\right] \leq \mathbb{P}\left[\hat{\sigma}_{t_{2}} \neq \mathrm{id}\right] \leq \mathbb{P}\left[\hat{\sigma}_{t_{2}} \neq \mathrm{id} \mid \sigma_{t_{1}} \in \tilde{B}_{N}\right]+\mathbb{P}\left[\sigma_{t_{1}} \notin \tilde{B}_{N}\right]$.

The second term is smaller than $2 \varepsilon$ from (38). The first term can be decomposed as follows

$$
\mathbb{P}\left[\hat{\sigma}_{t_{2}} \neq \mathrm{id} \mid \sigma_{t_{1}} \in \tilde{B}_{N}\right] \leq \mathbb{P}\left[\hat{\sigma}_{t_{2}} \neq \hat{\sigma}_{t_{2}}^{\min } \mid \sigma_{t_{1}} \in \tilde{B}_{N}\right]+\mathbb{P}\left[\hat{\sigma}_{t_{2}}^{\min } \neq \mathrm{id}\right] .
$$

Using a stochastic coupling with equilibrium we see that there exists $C>0$ such that

$$
\mathbb{P}\left[\hat{\sigma}_{t_{2}}^{\min } \neq \mathrm{id}\right] \leq \hat{\pi}_{N}\left(\mathcal{S}_{N} \backslash\{\mathrm{id}\}\right) \leq C N^{-1} .
$$

where the last estimate can be deduced from Lemma 11. The other contribution is bounded using the squeezing argument in (36): if $\hat{f}_{N, k}$ are the eigenfunctions corresponding to $\hat{p}$ we have

$$
\mathbb{P}\left[\hat{\sigma}_{t_{2}} \neq \hat{\sigma}_{t_{2}}^{\min } \mid \sigma_{t_{1}}\right] \leq\left(\sum_{k=1}^{N} \frac{\hat{f}_{N, k}\left(h_{k}\left(\sigma_{t_{1}}\right)\right)-\hat{f}_{N, k}\left(h_{k}(\mathrm{id})\right)}{\delta_{\min }\left(\hat{f}_{N, k}\right)}\right) e^{-\varepsilon N\left(\hat{\varrho}+\gamma_{N}\right)} .
$$

If $\sigma_{t_{1}} \in \tilde{B}_{N}$, then the first factor in the r.h.s. is bounded above by $N^{C \sqrt{N}}$ for some constant $C$, while the second term is smaller than $e^{-\varepsilon N / 2}$. This allows to conclude the proof.

4.6. Proof of Theorem 3. In this subsection, we present the modifications needed to obtain the mixing time starting from some general sequence of initial conditions $\left(\xi_{N}, N \geqslant 1\right)$ satisfying (10). We focus here on the case $\alpha>0$ since $\alpha=0$ is much simpler and can be immediately adapted from the material in Section 6.2. We denote by $\left(h_{t}^{\xi_{N}}, t \geqslant 0\right)$ the associated evolving height function, and we update the notation (31) to fit the initial condition

$$
L_{N, k}(t):=\ell_{N, k}\left(h_{t}^{\xi_{N}}\right) \text { and } R_{N, k}(t):=r_{N, k}\left(h_{t}^{\xi_{N}}\right)
$$

Let $(\rho(t, x), t \geqslant 0, x \in(0,1))$ be the hydrodynamic limit obtained in Theorem 4 . Recall the definition of $t_{\rho_{0}}(29)$, and set

$$
\ell_{\rho}(t):=\inf \{x \in[0,1]: \rho(t, x)>0\}, \quad r_{\rho}(t):=\sup \{x \in[0,1]: \rho(t, x)<1\} .
$$

Note that for $t \geq t_{\rho_{0}}$ we have $\ell_{\rho}(t)=r_{\rho}(t)=1-\alpha$ (inf and sup have to be interpreted as essential extrema here, since $\rho(t, \cdot)$ is defined in $\left.L^{\infty}\right)$. We have to prove the following generalization of Proposition 9. 
Proposition 13. Let $p \in(1 / 2,1]$. For any $t \geqslant 0$, we have the following convergence in probability

$$
\begin{aligned}
& \lim _{N \rightarrow \infty} N^{-1} L_{N, k}\left(\frac{N t}{p-q}\right)=\ell_{\rho}(t) \wedge(\ell+t), \\
& \lim _{N \rightarrow \infty} N^{-1} R_{N, k}\left(\frac{N t}{p-q}\right)=r_{\rho}(t) \vee(r-t) .
\end{aligned}
$$

The asserted mixing time of Theorem 3 is nothing but the first time $t \geq 0$ at which the limits obtained in this proposition reach the value $1-\alpha$. This being given, the proof presented in Subsection 4.3 works almost verbatim upon replacing $t_{\alpha, N}$ by

$$
t_{N}=\frac{N}{p-q} \max \left(t_{\rho}, 1-\alpha-\ell, r-1+\alpha\right) .
$$

Indeed, the lower bound follows as a corollary of Proposition 13 and Lemma 11. Regarding the upper bound in the case $p<1$, we first notice that

$$
P_{t}^{\xi_{N}}-\pi_{N, k}=\sum_{\xi^{\prime} \in \Omega_{N, k}} \pi_{N, k}\left(\xi^{\prime}\right)\left(P_{t}^{\xi_{N}}-P_{t}^{\xi^{\prime}}\right)
$$

so that

$$
\left\|P_{t}^{\xi_{N}}-\pi_{N, k}\right\|_{T V} \leqslant \pi_{N, k}\left(\Omega_{N, k} \backslash A_{N, k}^{\varepsilon}\right)+\sup _{\xi^{\prime} \in A_{N, k}^{\varepsilon}}\left\|P_{t}^{\xi_{N}}-P_{t}^{\xi^{\prime}}\right\|_{T V} .
$$

The first term goes to 0 by Lemma 11 . Using the monotonicity under the grand coupling $\mathbb{P}$ at the second line, we bound the second term as follows

$$
\begin{aligned}
\sup _{\xi^{\prime} \in A_{N, k}^{\varepsilon}}\left\|P_{t}^{\xi_{N}}-P_{t}^{\xi^{\prime}}\right\|_{T V} & \leqslant \sup _{\xi^{\prime} \in A_{N, k}^{\varepsilon}} \mathbb{P}\left[h_{t}^{\xi_{N}} \neq h_{t}^{\xi^{\prime}}\right] \\
& \leqslant \mathbb{P}\left[h_{t}^{\xi_{N}} \neq h_{t}^{\vee}\right]+\mathbb{P}\left[h_{t}^{\xi_{N}} \neq h_{t}^{\wedge_{N, k}^{\varepsilon}}\right] .
\end{aligned}
$$

Thus it suffices to show that both terms on the r.h.s. vanish when $t=$ $t_{N}+\delta N$, for any $\delta>0$ (notice that $t_{N}$ may be negligible compared to $N$ ). We write

$$
\begin{aligned}
\mathbb{P}\left[h_{t_{N}+\delta N}^{\xi_{N}} \neq h_{t_{N}+\delta N}^{\vee}\right] & \leqslant \mathbb{P}\left[h_{t_{N}+\delta N}^{\xi_{N}} \neq h_{t_{N}+\delta N}^{\vee} \mid h_{t_{N}}^{\xi_{N}} \in A_{N, k}^{\varepsilon}\right] \\
& +\mathbb{P}\left[h_{t_{N}}^{\xi_{N}} \notin A_{N, k}^{\varepsilon}\right]
\end{aligned}
$$

as well as

$$
\begin{aligned}
& \mathbb{P}\left[h_{t_{N}+\delta N}^{\xi_{N}} \neq h_{t_{N}+\delta N}^{\wedge \varepsilon_{N, k}^{\varepsilon}}\right] \leqslant \mathbb{P}\left[h_{t_{N}+\delta N}^{\xi_{N}} \neq h_{t_{N}+\delta N}^{\wedge_{N, k}^{\varepsilon}} \mid h_{t_{N}}^{\xi_{N}}, h_{t_{N}}^{\wedge_{N, k}^{\varepsilon}} \in A_{N, k}^{\varepsilon}\right] \\
&+\mathbb{P}\left[h_{t_{N}}^{\xi_{N}} \notin A_{N, k}^{\varepsilon}\right]+\mathbb{P}\left[h_{t_{N}}^{\wedge_{N, k}^{\varepsilon}} \notin A_{N, k}^{\varepsilon}\right] . \\
& \text { imsart-aop ver. 2014/10/16 file: AOP1290.tex date: May 9, } 2019
\end{aligned}
$$


From there, we can apply the same reasoning as in Subsection 4.3 to show that these two terms go to 0 as $N$ goes to $\infty$. This concludes the proof of Theorem 3 when $p<1$. To treat the case $p=1$, one simply has to adapt the arguments presented in Subsection 4.5.

5. Hydrodynamic limit. In this section, we speed up the jump rates by a factor $N /(p-q)$ in order to simplify the notations. The previous notation $\eta^{\xi_{N}}(t N /(p-q), x)$ now becomes $\eta(t, x)$.

The theory of solutions of the Burgers equation with zero-flux boundary conditions was developed in Bürger, Frid and Karlsen [8]. As it is shown in [18], the unique solution of this PDE coincides with the unique entropy solution of the Burgers equation with appropriate Dirichlet boundary conditions:

$$
\left\{\begin{array}{l}
\partial_{t} \rho=-\partial_{x}(\rho(1-\rho)), \quad t>0, x \in(0,1) \\
\rho(t, 0)=0, \quad \rho(t, 1)=1 \\
\rho(0, \cdot)=\rho_{0}(\cdot)
\end{array}\right.
$$

Therefore, we only need to prove convergence towards the latter object.

REMARK 14. As it is explained in [18], the particle system with zeroflux boundary conditions could essentially be obtained from the system on the whole line $\mathbb{Z}$ where we place only particles after site $N$ (density equal to 1) and no particle before site 0 (density equal to 0 ): this provides a heuristic explanation for our Dirichlet boundary conditions above.

The precise definition of the entropy solution of (39) is the following. Hereafter $\langle f, g\rangle$ denotes the $L^{2}([0,1], d y)$ inner product of $f$ and $g$.

DeFinition 15. Let $\rho_{0} \in L^{\infty}([0,1], d y)$. We say that a function $(\rho(t, y), t \geqslant 0, y \in$ $[0,1]) \in L^{\infty}\left(\mathbb{R}_{+} \times[0,1], d t \otimes d y\right)$ is an entropy solution of the inviscid Burgers equation (39) if for all $\kappa \in[0,1]$ and all $\varphi \in \mathcal{C}_{c}^{\infty}\left(\mathbb{R}_{+} \times \mathbb{R}, \mathbb{R}_{+}\right)$, we have

$$
\begin{aligned}
& \int_{0}^{\infty}\left(\left\langle\partial_{t} \varphi(t, \cdot),(\rho(t, \cdot)-\kappa)^{ \pm}\right\rangle+\left\langle\partial_{y} \varphi(t, \cdot), h^{ \pm}(\rho(t, \cdot), \kappa)\right\rangle\right. \\
& \left.\quad+\varphi(t, 0)(0-\kappa)^{ \pm}+\varphi(t, 1)(1-\kappa)^{ \pm}\right) d t+\left\langle\varphi(0, \cdot),(\rho(0, \cdot)-\kappa)^{ \pm}\right\rangle \geqslant 0,
\end{aligned}
$$

where $h^{+}(r, \kappa)=1_{\mathbb{R}_{+}}(r-\kappa)(r(1-r)-\kappa(1-\kappa))$ and $h^{-}(r, \kappa)=h^{+}(\kappa, r)$.

For any given initial condition $\rho_{0} \in L^{\infty}([0,1])$, there exists a unique entropy solution to the inviscid Burgers equation with Dirichlet boundary 
conditions, see Vovelle [31]. Let us mention that the original construction of entropy solutions is due to Bardos, Le Roux and Nédélec [2] in the BV setting, and is extended to the $L^{\infty}$ setting by Otto [28]. One should notice that the solution does not necessarily satisfy the boundary conditions but instead, satisfies the so-called BLN conditions. In particular, we do not expect the solution to be equal to 1 at $x=1$ for short times when it starts from $\wedge$ : indeed, it takes a macroscopic time for the rightmost particle to reach the right boundary and therefore the density of particles at $x=1$ remains null for a while.

Let $\left(\xi_{N}\right)_{N} \geqslant 1$ be a sequence of initial conditions and let $h\left(\xi_{N}\right)$ be the associated sequence of height functions. Observe that $\left(u_{0}^{N}(x):=\frac{1}{N} h\left(\xi_{N}\right)(x N), x \in\right.$ $[0,1])$ is 1-Lipschitz so that it is tight in $\mathcal{C}([0,1])$.

Lemma 16. For any choice of initial conditions, the sequence of processes $\rho^{N}$ and $u^{N}$ are $\mathbb{C}$-tight in $\mathbb{D}([0, \infty), \mathcal{M})$ and $\mathbb{D}([0, \infty), \mathcal{C}([0,1]))$ respectively.

Proof. The arguments are standard so we only give a sketch of proof. To prove tightness of $\rho^{N}$ it suffices to show that

$$
\varlimsup_{\delta \downarrow 0} \varlimsup_{N \rightarrow \infty} \mathbb{E}\left[\sup _{s, t \leqslant T,|t-s| \leqslant \delta}\left|\left\langle\rho_{t}^{N}-\rho_{s}^{N}, \varphi\right\rangle\right|\right]=0,
$$

for all $\varphi \in \mathcal{C}^{\infty}([0,1])$. To that end, we write

$$
\left\langle\rho_{t}^{N}, \varphi\right\rangle-\left\langle\rho_{s}^{N}, \varphi\right\rangle=\int_{s}^{t} L^{N}\left\langle\rho_{r}^{N}, \varphi\right\rangle d r+M_{s, t}^{N},
$$

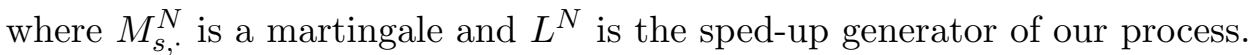
Then, it is simple to bound the two terms on the r.h.s, see for instance [29, Lemma 4.1].

Regarding the tightness of the sequence $u^{N}$, we first observe that for all $t \geqslant 0$, the profiles $y \mapsto u^{N}(t, y)$ are 1-Lipschitz. Furthermore, for every $y \in[0,1]$, the process $t \mapsto u^{N}(t, y)$ makes jumps of size at most $2 / N$ and at rate at most $N /(p-q)$. Using the moments formula for the Poisson r.v., we deduce that for all $m \geqslant 1$ we have

$$
\sup _{y \in[0,1]} \mathbb{E}\left[\left|u^{N}(t, y)-u^{N}(s, y)\right|^{m}\right]^{\frac{1}{m}} \lesssim \frac{|t-s|^{\frac{1}{m}}}{N^{\frac{m-1}{m}}}+|t-s|,
$$

uniformly over all $s, t \geqslant 0$ and all $N \geqslant 1$. Then, one defines $\bar{u}^{N}$ as the continuous-time interpolation of $u^{N}$ taken at all times $t \in \mathbb{Z} / N$. It is straightforward to check that $\bar{u}^{N}$ is tight using the estimate already obtained on $u^{N}$. 
To conclude, we only need to check that $u^{N}$ and $\bar{u}^{N}$ are uniformly close on any given compact space-time set: this can be done by bounding the moments of the supremum of $\left|u^{N}-\bar{u}^{N}\right|$ on boxes of size $1 / N \times 1 / N$ and then summing over a covering of the given compact set into such boxes, see for instance [5, Lemma 4.7].

Below, we will consider an initial probability measure $\iota_{N}$ on $\cup_{k} \Omega_{N, k}^{0}$ that satisfies the following assumption.

Assumption 1. There exists a piecewise constant function $f:[0,1] \rightarrow$ $[0,1]$ such that for all $N \geqslant 1, \iota_{N}=\otimes_{x=1}^{N} \operatorname{Be}(f(x / N))$ where $\operatorname{Be}(c)$ denotes the Bernoulli \pm 1 distribution with parameter $c$.

The main step of the proof of Theorem 4 consists in establishing the hydrodynamic limit starting from elementary initial conditions.

TheOREm 5. We work under Assumption 1 and we let $f$ be the density appearing therein. The sequence of empirical densities $\rho^{N}$ converges in probability in the Skorohod space $\mathbb{D}([0, \infty), \mathcal{M})$ to the deterministic process $\rho(t, d y)=\rho(t, y) d y$ where $(\rho(t, y), y \in[0,1], t \geqslant 0)$ is the entropy solution of (39) with initial condition $\rho(0, \cdot)=f(\cdot)$.

Given this result, we turn to the proof of Theorem 4 .

Proof of Theorem 4. Since we assume that $\rho_{0}^{N}$ converges weakly to $\rho_{0}$, it is not difficult to deduce that

$$
\lim _{N \rightarrow \infty} \sup _{y \in[0,1]}\left|u_{0}^{N}(y)-u_{0}(y)\right|=0
$$

where $u_{0}(y)=\int_{0}^{y}\left(2 \rho_{0}(x)-1\right) d x$. Thanks to Lemma 16 , we only need to check that any limiting points $\rho$ of $\rho^{N}$ is the entropy solution of the Burgers equation (39) starting from $\rho_{0}$ and that any limiting point $u$ of $u^{N}$ is its integrated version. The latter property is actually simple to establish once we know that $\rho^{N}$ converges to $\rho$ so we concentrate on this convergence.

In the case where the sequence of initial conditions $\rho_{0}^{N}$ satisfies Assumption 1, Theorem 5 yields the convergence of $\rho^{N}$ towards the entropy solution of (39). To extend the scope of this convergence result to a general sequence of initial conditions $\xi_{N}$, we proceed by approximation. Set $\alpha=\left(1+u_{0}(1)\right) / 2$, and recall that $u_{0}$ is 1 Lipschitz so that $\rho_{0}(x)$ is almost everywhere in $[0,1]$. Fix $\epsilon>0$. One can find two profiles $u_{0}^{+, \epsilon}, u_{0}^{-, \epsilon}$ which are 1-Lipschitz, start from 0 at 0 , are piecewise affine and satisfy the inequalities (see Figure 2): 


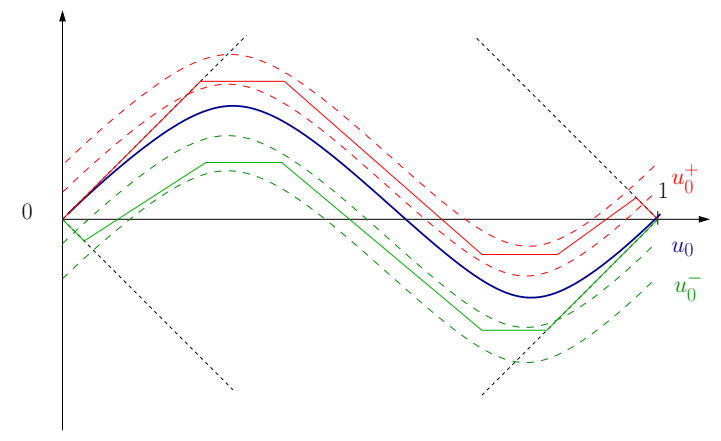

FIG 2. How to bound $u_{0}$ by two piecewise affine functions: an important feature of the construction is that we force the slope of $u_{0}^{ \pm}$to be \pm 1 near the boundary so that the inequality still holds with high probability when the corresponding microscopic height functions are compared.

$$
\begin{aligned}
u_{0}(y)-2 \epsilon & \leqslant u_{0}^{-, \epsilon}(y) \leqslant\left(u_{0}(y)-\epsilon\right) \vee(-y) \vee(y-2+2 \alpha), \\
\left(u_{0}(y)+\epsilon\right) \wedge y \wedge(2 \alpha-y) & \leqslant u_{0}^{+, \epsilon}(y) \leqslant u_{0}(y)+2 \epsilon,
\end{aligned}
$$

and are such that $\left\|\rho_{0}^{ \pm, \epsilon}-\rho_{0}\right\|_{L^{1}}$ goes to 0 as $\epsilon \downarrow 0$, where $\rho_{0}^{ \pm, \epsilon}:=\left(\partial_{y} u_{0}^{ \pm, \epsilon}+1\right) / 2$. Then, for every $N \geqslant 1$ we consider three initial configurations of the ASEP: one is given by $\xi_{N}$, the two others $\xi_{N}^{ \pm, \epsilon}$ are random elements in $\cup_{k} \Omega_{N, k}^{0}$ with law $\otimes_{x=1}^{N} \operatorname{Be}\left(\rho_{0}^{ \pm, \epsilon}(x / N)\right)$. We couple these three ASEP in such a way that the order on the height functions is preserved by the dynamics (similarly as in our grand coupling). Equation (42) ensures that the probability of the event

$$
h\left(\xi_{N}^{-, \epsilon}\right)(t=0, \cdot) \leqslant h\left(\xi_{N}\right)(t=0, \cdot) \leqslant h\left(\xi_{N}^{+, \epsilon}\right)(t=0, \cdot),
$$

goes to 1 as $N \rightarrow \infty$. Therefore, the probability that these inequalities happen at all times $t \geq 0$ goes to 1 as well.

Our convergence result applies to $\rho_{N}^{ \pm, \epsilon}$ (which are the empirical densities associated to $\left.h\left(\xi_{N}^{ \pm, \epsilon}\right)\right)$ : the limits $\rho^{ \pm, \epsilon}$ are the solutions of (39) starting from $\rho_{0}^{ \pm, \epsilon}$. Let us denote by $u^{ \pm, \epsilon}$ the associated integrated solutions. Our coupling ensures that any limit point $u$ of the tight sequence $u^{N}$ lies in between these two integrated solutions. By the $L^{1}$ contractivity [27, Th. 7.28] of the solution to (39), one deduces that as $\epsilon \downarrow 0, u^{ \pm, \epsilon}$ converges to the integrated solution of (39) starting from $u_{0}$, thus $u$ coincides with this solution and this concludes the proof. 
We are now left with proving Theorem 5 . To that end, it suffices to show that any limit point of the tight sequence $\rho^{N}$ satisfies the entropy inequalities of Definition 15. The usual trick that makes the contant $\kappa$ appear in these inequalities is to couple $\eta$ with another particle system $\zeta$ which is stationary with distribution $\otimes_{x=1}^{N} \operatorname{Be}(\kappa)$. Actually, our boundary conditions complicate the proof and it will be convenient to consider another particle system which evolves according to the same dynamics but on the whole line $\mathbb{Z}$.

More precisely, we will consider the process $(\eta, \zeta, \hat{\eta}, \hat{\zeta})$ where each element of the quadruplet is a process that lives in $\{0,1\}^{\mathbb{Z}}$ and such that the following holds. The restriction of $\eta$ to $\llbracket 1, N \rrbracket$ is a Markov process evolving according to the ASEP dynamics considered from the beginning of this paper, while the restriction of $\eta$ to $\mathbb{Z} \backslash \llbracket 1, N \rrbracket$ remains constant. The process $\zeta$ remains constant outside $\llbracket 1, N \rrbracket$, while in $\llbracket 1, N \rrbracket$ it undergoes the same dynamics as $\eta$ except that at site 1 if there is no particle, then a particle is created at rate $N(2 p-1) \kappa /(p-q)$ and at site $N$, if there is a particle, then it is removed at rate $N(2 p-1)(1-\kappa) /(p-q)$. Regarding $\hat{\eta}$ and $\hat{\zeta}$, they evolve according to the $\operatorname{ASEP}(p, q)$ on the whole line $\mathbb{Z}$ without any boundary effect: the dynamics is translation invariant.

Let us now explain how the processes are coupled. For all pairs of consecutive sites in $\llbracket 1, N \rrbracket$, we make the jumps simultaneous for the four particle systems. For all pairs of consecutive sites in $\mathbb{Z} \backslash \llbracket 2, N-1 \rrbracket$, we make the jumps simultaneous for $\hat{\eta}$ and $\hat{\zeta}$. Let us point out that each of these processes is Markov. Instead of writing down the generator $\tilde{\mathcal{L}}$ of the quadruplet acting on a general test function, we restrict to test functions involving only two of the four processes. We set $b(x, y):=x(1-y)$. The generator acting on $\eta, \zeta$ is given by

$$
\tilde{\mathcal{L}} f(\eta, \zeta)=\tilde{\mathcal{L}}^{\text {bulk }} f(\eta, \zeta)+\tilde{\mathcal{L}}^{\text {bdry }} f(\eta, \zeta)
$$

where

$$
\tilde{\mathcal{L}}^{\text {bulk }} f(\eta, \zeta)=\frac{N}{p-q} \sum_{k, \ell=1}^{N}\left(p 1_{\{\ell-k=1\}}+q 1_{\{k-\ell=1\}}\right) \mathcal{G}_{k, \ell}(\eta, \zeta),
$$

where

$$
\begin{aligned}
\mathcal{G}_{k, \ell}(\eta, \zeta) & :=(b(\eta(k), \eta(\ell)) \wedge b(\zeta(k), \zeta(\ell)))\left(f\left(\eta^{k, \ell}, \zeta^{k, \ell}\right)-f(\eta, \zeta)\right) \\
& +(b(\eta(k), \eta(\ell))-b(\eta(k), \eta(\ell)) \wedge b(\zeta(k), \zeta(\ell)))\left(f\left(\eta^{k, \ell}, \zeta\right)-f(\eta, \zeta)\right) \\
& +(b(\zeta(k), \zeta(\ell))-b(\eta(k), \eta(\ell)) \wedge b(\zeta(k), \zeta(\ell)))\left(f\left(\eta, \zeta^{k, \ell}\right)-f(\eta, \zeta)\right)
\end{aligned}
$$

and, using the notation $\zeta \pm \delta_{k}$ to denote the particle configuration which coincides with $\zeta$ everywhere except at site $k$ where the occupation is taken 
to be $\zeta(k) \pm 1$

$$
\begin{aligned}
\tilde{\mathcal{L}}^{\mathrm{bdry}} f(\eta, \zeta) & =\frac{N}{p-q}(2 p-1) \kappa(1-\zeta(1))\left(f\left(\eta, \zeta+\delta_{1}\right)-f(\eta, \zeta)\right) \\
& +\frac{N}{p-q}(2 p-1)(1-\kappa) \zeta(N)\left(f\left(\eta, \zeta-\delta_{N}\right)-f(\eta, \zeta)\right) .
\end{aligned}
$$

The generator acting on $\hat{\eta}, \hat{\zeta}$ is given by

$$
\tilde{\mathcal{L}} f(\hat{\eta}, \hat{\zeta})=\frac{N}{p-q} \sum_{k, \ell \in \mathbb{Z}}\left(p 1_{\{\ell-k=1\}}+q 1_{\{k-\ell=1\}}\right) \mathcal{G}_{k, \ell}(\hat{\eta}, \hat{\zeta}) .
$$

Let us also provide the expression of the generator acting on $\eta, \hat{\eta}$ :

$$
\begin{aligned}
\tilde{\mathcal{L}} f(\eta, \hat{\eta}) & =\frac{N}{p-q} \sum_{k, \ell=1}^{N}\left(p 1_{\{\ell-k=1\}}+q 1_{\{k-\ell=1\}}\right) \mathcal{G}_{k, \ell}(\eta, \hat{\eta}) \\
& +\frac{N}{p-q} \sum_{k, \ell \in \mathbb{Z} \backslash \rrbracket 2, N-1 \rrbracket}\left(p 1_{\{\ell-k=1\}}+q 1_{\{k-\ell=1\}}\right)\left(f\left(\eta, \hat{\eta}^{k, \ell}\right)-f(\eta, \hat{\eta})\right) .
\end{aligned}
$$

Let us introduce an initial condition that will be useful in the sequel. Let $\iota_{N}$ be a measure on $\cup_{k} \Omega_{N, k}^{0}$ that satisfies Assumption 1. Let the restriction of $\eta_{0}$ to $\llbracket 1, N \rrbracket$ start with law $\iota_{N}$ and let the restriction of $\zeta_{0}$ to $\llbracket 1, N \rrbracket$ start with law given by a product of Bernoulli measures $\mathrm{Be}(\kappa)$. We also let $\eta_{0}(x)=\zeta_{0}(x)=0$ for all $x \in \mathbb{Z} \backslash \llbracket 1, N \rrbracket$. These two initial conditions are coupled in the following way: for every $x \in \llbracket 1, N \rrbracket$, we have $\eta_{0}(x) \geqslant \zeta_{0}(x)$ if and only if $f(x / N) \geqslant \kappa$, where $f$ is the density arising in Assumption 1. Additionally, we set $\hat{\eta}_{0}(x)=\eta_{0}(x)$ for all $x \in \mathbb{Z}$. Finally, we set $\hat{\zeta}_{0}(x)=\zeta_{0}(x)$ for all $x \in \llbracket 1, N \rrbracket$, and we draw the remaining values of $\hat{\zeta}_{0}(\cdot)$ according to an independent sequence of $\operatorname{Be}(\kappa)$. The law of the quadruplet starting from this initial condition will be denoted by $\mathbb{Q}_{\iota_{N}, \kappa}^{N}$.

The first step consists in establishing the entropy inequalities at the microscopic level. Recall the notation $b(x, y)=x(1-y)$. We define a function $F_{k, \ell}$ acting on a pair of particle systems on $\mathbb{Z}$ as follows. We set $F_{k, \ell}(\eta, \zeta)=1$ if $\eta(k) \geqslant \zeta(k)$ and $\eta(\ell) \geqslant \zeta(\ell)$; otherwise we set $F_{k, \ell}(\eta, \zeta)=0$. Moreover we set

$H^{+}(\eta, \zeta)=(b(\eta(1), \eta(0))-b(\zeta(1), \zeta(0))) F_{1,0}(\eta, \zeta), \quad H^{-}(\eta, \zeta)=H^{+}(\zeta, \eta)$.

We also let

$$
\langle f, g\rangle_{N}:=\frac{1}{N} \sum_{k \in \mathbb{Z}} f(k) g(k)
$$

imsart-aop ver. 2014/10/16 file: AOP1290.tex date: May 9, 2019 
Lemma 17 (Microscopic inequalities). We work under Assumption 1. For all $\varphi \in \mathcal{C}_{c}^{\infty}\left(\mathbb{R}_{+} \times \mathbb{R}, \mathbb{R}_{+}\right)$, all $\delta>0$ and all $\kappa \in[0,1]$, we have $\lim _{N \rightarrow \infty} \mathbb{Q}_{\iota_{N}, \kappa}^{N}\left(I_{\text {micro }} \geqslant-\right.$ $\delta)=1$ where $I_{\text {micro }}$ denotes either

$$
\begin{aligned}
& \int_{0}^{\infty}\left(\left\langle\partial_{t} \varphi(t, \cdot),(\eta(t, \cdot)-\zeta(t, \cdot))^{ \pm}\right\rangle_{N}+\left\langle\partial_{x} \varphi(t, \cdot), H^{ \pm}(\tau . \eta(t), \tau \cdot \zeta(t))\right\rangle_{N}\right. \\
& \left.+\left((0-\kappa)^{ \pm} \varphi(t, 0)+(1-\kappa)^{ \pm} \varphi(t, 1)\right)\right) d t+\left\langle\varphi(0, \cdot),(\eta(0, \cdot)-\zeta(0, \cdot))^{ \pm}\right\rangle_{N}
\end{aligned}
$$

or

$$
\begin{aligned}
\int_{0}^{\infty}( & \left.\left\langle\partial_{t} \varphi(t, \cdot),(\hat{\eta}(t, \cdot)-\hat{\zeta}(t, \cdot))^{ \pm}\right\rangle_{N}+\left\langle\partial_{x} \varphi(t, \cdot), H^{ \pm}(\tau \cdot \hat{\eta}(t), \tau \cdot \hat{\zeta}(t))\right\rangle_{N}\right) d t \\
& +\left\langle\varphi(0, \cdot),(\hat{\eta}(0, \cdot)-\hat{\zeta}(0, \cdot))^{ \pm}\right\rangle_{N} \cdot
\end{aligned}
$$

Proof. This is similar to Lemma 2.10 in [18]. Let us recall the main steps here in the case of $(\eta, \zeta)$ : the case of $(\hat{\eta}, \hat{\zeta})$ is simpler since we don't have to deal with the boundary terms. First of all, we set

$$
\begin{aligned}
B_{t}=\int_{0}^{t}\left(\left\langle\partial_{s} \varphi(s, \cdot),(\eta(s, \cdot)-\zeta(s, \cdot))^{ \pm}\right\rangle_{N}\right. & \left.+\tilde{\mathcal{L}}\left\langle\varphi(s, \cdot),(\eta(s, \cdot)-\zeta(s, \cdot))^{ \pm}\right\rangle_{N}\right) d s \\
& +\left\langle\varphi(0, \cdot),(\eta(0, \cdot)-\zeta(0, \cdot))^{ \pm}\right\rangle_{N} .
\end{aligned}
$$

By definition of the generator, we have the identity

$$
\left\langle\varphi(t, \cdot),(\eta(t, \cdot)-\zeta(t, \cdot))^{ \pm}\right\rangle_{N}=B_{t}+M_{t},
$$

where $M$ is a mean-zero martingale. A long calculation shows that the term in $B_{t}$ involving the generator is bounded by

$$
\left\langle\partial_{x} \varphi(s, \cdot), H^{ \pm}(\tau \cdot \eta(s), \tau . \zeta(s))\right\rangle_{N}+(0-\kappa)^{ \pm} \varphi(s, 0)+(1-\kappa)^{ \pm} \varphi(s, 1),
$$

up to a negligible term of order $1 / N$. Moreover, the Burkholder-Davis-Gundy inequality allows one to bound the moments of the martingale and to show that they vanish as $N \rightarrow \infty$. Using the fact that $\varphi$ is compactly supported, one gets $B_{t}=-M_{t}$ for $t$ large enough. The assertion of the lemma follows by putting everything together.

The next step consists in replacing the microscopic quantities by averages on boxes of size $\ell$. We denote by $T_{\ell}(k):=\llbracket k-\ell, k+\ell \rrbracket$ and we set

$$
\mathscr{M}_{T_{\ell}(k)} f=\frac{1}{2 \ell+1} \sum_{i \in T_{\ell}(k)} f(i),
$$


for any map $f: \mathbb{Z} \rightarrow \mathbb{R}$. The invariance by translation of the dynamics of $(\hat{\eta}, \hat{\zeta})$ ensures that for any $A, T>0$ and for any initial condition $\left(\hat{\eta}_{0}, \hat{\zeta}_{0}\right)$ such that $\hat{\zeta}_{0}$ is a product of $\operatorname{Be}(\kappa)$, one has

$$
\begin{aligned}
& \lim _{\ell \rightarrow \infty} \lim _{N \rightarrow \infty} \mathbb{Q}^{N}[ \int_{0}^{T} \frac{1}{N} \sum_{k=-A N}^{A N} \mid \mathscr{M}_{T_{\ell}(k)}(\hat{\eta}(t)-\hat{\zeta}(t))^{ \pm} \\
&\left.-\left(\mathscr{M}_{T_{\ell}(k)} \hat{\eta}(t)-\kappa\right)^{ \pm} \mid d t\right]=0, \\
& \lim _{\ell \rightarrow \infty} \lim _{N \rightarrow \infty} \mathbb{Q}^{N}\left[\int_{0}^{T} \frac{1}{N} \sum_{k=-A N}^{A N} \mid \mathscr{M}_{T_{\ell}(k)} H^{ \pm}(\hat{\eta}(t), \hat{\zeta}(t))\right. \\
&\left.-h^{ \pm}\left(\mathscr{M}_{T_{\ell}(k)} \hat{\eta}(t), \kappa\right) \mid d t\right]=0,
\end{aligned}
$$

see the arguments on pp.426-427 of Rezakhanlou [29]. These arguments do not apply anymore to $(\eta, \zeta)$. However the next lemma shows that $\eta-\hat{\eta}$ and $\zeta-\hat{\zeta}$ are small in the bulk of the lattice, and therefore, one deduces that (43) also holds with $(\hat{\eta}, \hat{\zeta})$ replaced by $(\eta, \zeta)$. In the lemma below, we let $\mathbb{Q}$ be the law of the dynamics starting from some deterministic initial condition $\left(\eta_{0}, \zeta_{0}, \hat{\eta}_{0}, \hat{\zeta}_{0}\right)$

Lemma 18. There exits a constant $C>0$ such that for all $\epsilon>0$, we have

$$
\lim _{N \rightarrow \infty} \sup _{\eta_{0}=\hat{\eta}_{0} \in\{0,1\}^{\mathbb{Z}}} \sup _{s \in[0, C \epsilon]} \mathbb{Q}\left[\frac{1}{N} \sum_{x \in[\epsilon N, N-\epsilon N]}|\eta(s, x)-\hat{\eta}(s, x)|\right]=0,
$$

and similarly with $(\zeta, \hat{\zeta})$.

This lemma is in the spirit of [1, Lemma 3.3].

Proof. Fix $\epsilon>0$. Let $\varphi$ be a function from $\mathbb{R}_{+} \times \mathbb{R}$ into $\mathbb{R}_{+}$such that:

1. $\varphi(t, x)=0$ as soon as $x \notin[\epsilon / 3,1-\epsilon / 3]$,

2. $\varphi(t, x)>c$ for all $(t, x) \in[0, C \epsilon] \times[\epsilon, 1-\epsilon]$ and for some $c, C>0$,

3. $\partial_{t} \varphi+2\left|\partial_{x} \varphi\right| \leqslant 0$.

Such a function exists. Take for instance $\varphi(t, x)=\Phi\left(6 t \epsilon^{-1}+q(x)\right)$ where $\Phi: \mathbb{R} \rightarrow[0,1]$ is smooth, non-increasing, equal to 1 on $\mathbb{R}_{-}$and to 0 on $[1, \infty)$, and $q: \mathbb{R} \mapsto[0,1]$ is equal to 0 on $[\epsilon, 1-\epsilon]$, to 1 on $(-\infty, \epsilon / 3] \cup[1-\epsilon / 3, \infty)$ and is such that $\left\|q^{\prime}\right\|_{\infty} \leqslant 3 \epsilon^{-1}$.

Since $\eta_{0}=\hat{\eta}_{0}$ and $\varphi$ vanishes on the boundaries, we have

$$
\left\langle\varphi(t, \cdot),\left(\eta_{t}-\hat{\eta}_{t}\right)^{ \pm}\right\rangle_{N}=I_{t}^{ \pm}+M_{t}^{ \pm}
$$


where $M_{t}^{ \pm}$is a martingale and

$$
I_{t}^{ \pm}=\int_{0}^{t}\left(\left\langle\partial_{s} \varphi(s, \cdot),\left(\eta_{s}-\hat{\eta}_{s}\right)^{ \pm}\right\rangle_{N}+\tilde{\mathcal{L}}\left\langle\varphi(s, \cdot),\left(\eta_{s}-\hat{\eta}_{s}\right)^{ \pm}\right\rangle_{N}\right) d s
$$

Using the computation in the proof of Lemma 17, we obtain

$$
\begin{aligned}
I_{t}^{ \pm} & \leqslant \int_{0}^{t}\left(\left\langle\partial_{s} \varphi(s, \cdot),\left(\eta_{s}-\hat{\eta}_{s}\right)^{ \pm}\right\rangle_{N}+\left\langle\partial_{x} \varphi(s, \cdot), H^{ \pm}\left(\tau . \eta_{s}, \tau \cdot \hat{\eta}_{s}\right)\right\rangle_{N}\right) d s \\
& +C_{\phi} N^{-1}
\end{aligned}
$$

where $C_{\phi}$ is a positive constant which depends only $\phi$ and could be made explicit (we will use the same notation for similar constant, as we do not believe it should yield confusion).

Let $H:=H^{+}+H^{-}$. By considering all possible configurations, we can check that

$$
\left|H\left(\tau_{k} \eta_{s}, \tau_{k} \hat{\eta}_{s}\right)\right| \leqslant\left|\eta_{s}(k)-\hat{\eta}_{s}(k)\right|+\left|\eta_{s}(k+1)-\hat{\eta}_{s}(k+1)\right| .
$$

Furthermore, we have

$$
\begin{aligned}
\left\langle\partial_{s} \varphi(s, \cdot),\left|\eta_{s}-\hat{\eta}_{s}\right|\right\rangle_{N} & =\left\langle\partial_{s} \varphi(s, \cdot), \frac{\left|\eta_{s}(\cdot)-\hat{\eta}_{s}(\cdot)\right|+\left|\eta_{s}(\cdot+1)-\hat{\eta}_{s}(\cdot+1)\right|}{2}\right\rangle_{N} \\
& +C_{\phi} N^{-1},
\end{aligned}
$$

uniformly over all $s \geqslant 0$ and all $N \geqslant 1$. Consequently, we get

$$
\begin{aligned}
I_{t}^{+}+I_{t}^{-} \leqslant & C_{\phi} N^{-1}+\int_{0}^{t}\left\langle\partial_{s} \varphi(s, \cdot)\right. \\
& \left.+2\left|\partial_{x} \varphi(s, \cdot)\right|, \frac{\left|\eta_{s}(\cdot)-\hat{\eta}_{s}(\cdot)\right|+\left|\eta_{s}(\cdot+1)-\hat{\eta}_{s}(\cdot+1)\right|}{2}\right\rangle_{N} d s \\
\leqslant & C_{\phi} N^{-1}
\end{aligned}
$$

uniformly over all $t$ in a compact set. Using the properties of the function $\varphi$, we deduce that uniformly over all $t \in[0, C \epsilon]$

$$
\begin{aligned}
& \mathbb{Q}\left[\frac{1}{N} \sum_{x \in[\epsilon N, N-\epsilon N]}|\eta(t, x)-\hat{\eta}(t, x)|\right] \\
& \leqslant \frac{1}{c} \mathbb{Q}\left[\left\langle\varphi(t, \cdot),\left|\eta_{t}-\hat{\eta}_{t}\right|\right\rangle_{N}\right] \leqslant \frac{1}{c} \mathbb{Q}\left[M_{t}^{+}+M_{t}^{-}\right]+C_{\phi} N^{-1} \leq C_{\phi} N^{-1},
\end{aligned}
$$

so that the statement of the lemma follows. 
Lemma 19. We work under Assumption 1. For all $\varphi \in \mathcal{C}_{c}^{\infty}\left(\mathbb{R}_{+} \times \mathbb{R}, \mathbb{R}_{+}\right)$, all $\delta>0$ and all $\kappa \in[0,1]$, we have $\lim _{\ell \rightarrow \infty} \lim _{N \rightarrow \infty} \mathbb{Q}_{\iota_{N}, \kappa}^{N}\left(I_{\text {meso }} \geqslant-\delta\right)=1$ where $I_{\text {meso }}$ is given by

$$
\begin{aligned}
& \int_{0}^{\infty}\left(\left\langle\partial_{t} \varphi(t, \cdot),\left(\mathscr{M}_{T_{\ell}(\cdot)} \eta(t)-\kappa\right)^{ \pm}\right\rangle_{N}+\left\langle\partial_{x} \varphi(t, \cdot), h^{ \pm}\left(\mathscr{M}_{T_{\ell}(\cdot)} \eta(t), \kappa\right)\right\rangle_{N}\right. \\
& \left.+\left((0-\kappa)^{ \pm} \varphi(t, 0)+(1-\kappa)^{ \pm} \varphi(t, 1)\right)\right) d t+\left\langle\varphi(0, \cdot),\left(\mathscr{M}_{T_{\ell}(\cdot)} \eta(0)-\kappa\right)^{ \pm}\right\rangle .
\end{aligned}
$$

Proof. From Lemma 17 and the smoothness of $\varphi$, we deduce that

$$
\lim _{\ell \rightarrow \infty} \lim _{N \rightarrow \infty} \mathbb{Q}_{\iota_{N}, \kappa}^{N}\left(J_{\text {micro }} \geqslant-\delta\right)=1
$$

where $J_{\text {micro }}$ is given by

$$
\begin{aligned}
& \int_{0}^{\infty}\left(\frac{1}{N} \sum_{k=1}^{N}\left(\partial_{t} \varphi(t, k) \mathscr{M}_{T_{\ell}(k)}(\eta(t)-\zeta(t))^{ \pm}+\partial_{x} \varphi(t, k) \mathscr{M}_{T_{\ell}(k)} H^{ \pm}(\eta(t), \zeta(t))\right)\right. \\
& \left.\quad+\left((0-\kappa)^{ \pm} \varphi(t, 0)+(1-\kappa)^{ \pm} \varphi(t, 1)\right)\right) d t
\end{aligned}
$$$$
+\frac{1}{N} \sum_{k=1}^{N} \varphi(0, k) \mathscr{M}_{T_{\ell}(k)}(r
$$

Since $\varphi$ is compactly supported, we can restrict the time integral to $[0, T]$ for some large enough $T>0$. The statement of the lemma follows if we are able to show that as $N \rightarrow \infty$ and $\ell \rightarrow \infty$ the $\mathbb{Q}_{\iota_{N}, \kappa}^{N}$-expectations of the following three quantities vanish

$$
\begin{gathered}
\int_{0}^{T} \frac{1}{N} \sum_{k=1}^{N}\left|\mathscr{M}_{T_{\ell}(k)}(\eta(t)-\zeta(t))^{ \pm}-\left(\mathscr{M}_{T_{\ell}(k)} \eta(t)-\kappa\right)^{ \pm}\right| d t \\
\int_{0}^{T} \frac{1}{N} \sum_{k=1}^{N}\left|\mathscr{M}_{T_{\ell}(k)} H^{ \pm}(\eta(t), \zeta(t))-h^{ \pm}\left(\mathscr{M}_{T_{\ell}(k)} \eta(t), \kappa\right)\right| d t \\
\frac{1}{N} \sum_{k=1}^{N}\left|\mathscr{M}_{T_{\ell}(k)}(\eta(0)-\zeta(0))^{ \pm}-\left(\mathscr{M}_{T_{\ell}(k)} \eta(0)-\kappa\right)^{ \pm}\right| .
\end{gathered}
$$

For the third one, it suffices to use the fact that the number of sign changes of $k \mapsto \eta(0, k)-\zeta(0, k)$ is uniformly bounded over $N \geqslant 1$ (this is a consequence of our coupling of the initial conditions). We now concentrate on the convergence of the second expression, since the convergence of the first follows from similar arguments. Fix $\epsilon>0$. Let $(\eta, \zeta, \hat{\eta}, \hat{\zeta})$ be the process defined previously in this section except that at every time $t_{i}=i C \epsilon, i \geqslant 1$, we 
reinitialise $\hat{\eta}$ and $\hat{\zeta}$ by letting them be equal to $\eta$ and $\zeta$ at this same time. We let $\mathbb{Q}^{N}$ be the law of the corresponding process. Then we write,

$$
\begin{aligned}
& \left|\mathscr{M}_{T_{\ell}(k)} H^{ \pm}(\eta(t), \zeta(t))-h^{ \pm}\left(\mathscr{M}_{T_{\ell}(k)} \eta(t), \kappa\right)\right| \\
& \leqslant\left|\mathscr{M}_{T_{\ell}(k)} H^{ \pm}(\eta(t), \zeta(t))-\mathscr{M}_{T_{\ell}(k)} H^{ \pm}(\hat{\eta}(t), \zeta(t))\right| \\
& \quad+\left|\mathscr{M}_{T_{\ell}(k)} H^{ \pm}(\hat{\eta}(t), \zeta(t))-\mathscr{M}_{T_{\ell}(k)} H^{ \pm}(\hat{\eta}(t), \hat{\zeta}(t))\right| \\
& \quad+\left|\mathscr{M}_{T_{\ell}(k)} H^{ \pm}(\hat{\eta}(t), \hat{\zeta}(t))-h^{ \pm}\left(\mathscr{M}_{T_{\ell}(k)} \hat{\eta}(t), \kappa\right)\right| \\
& \quad+\left|h^{ \pm}\left(\mathscr{M}_{T_{\ell}(k)} \hat{\eta}(t), \kappa\right)-h^{ \pm}\left(\mathscr{M}_{T_{\ell}(k)} \eta(t), \kappa\right)\right|,
\end{aligned}
$$

and we bound separately the contributions coming from the terms arising on the right hand side. Notice that (43) still holds for $(\hat{\eta}, \hat{\zeta})$ as long as we apply it to interval of times of the form $\left[t_{i}, t_{i+1}\right.$ ) (since our modified dynamics coincides with the original one on these intervals). Therefore, for every $i \geqslant 0$

$\lim _{\ell \rightarrow \infty} \lim _{N \rightarrow \infty} \mathbb{Q}^{N}\left[\int_{t_{i}}^{t_{i+1}} \frac{1}{N} \sum_{k=1}^{N}\left|\mathscr{M}_{T_{\ell}(k)} H^{ \pm}(\hat{\eta}(t), \hat{\zeta}(t))-h^{ \pm}\left(\mathscr{M}_{T_{\ell}(k)} \hat{\eta}(t), \kappa\right)\right| d t\right]=0$,

and we deduce that

$\lim _{\ell \rightarrow \infty} \lim _{N \rightarrow \infty} \mathbb{Q}^{N}\left[\int_{0}^{T} \frac{1}{N} \sum_{k=1}^{N}\left|\mathscr{M}_{T_{\ell}(k)} H^{ \pm}(\hat{\eta}(t), \hat{\zeta}(t))-h^{ \pm}\left(\mathscr{M}_{T_{\ell}(k)} \hat{\eta}(t), \kappa\right)\right| d t\right]=0$.

On the other hand, as long as $\ell \leqslant \epsilon N$ we write

$$
\begin{aligned}
& \int_{0}^{T} \frac{1}{N} \sum_{k=1}^{N}\left|\mathscr{M}_{T_{\ell}(k)} H^{ \pm}(\eta(t), \zeta(t))-\mathscr{M}_{T_{\ell}(k)} H^{ \pm}(\hat{\eta}(t), \zeta(t))\right| d t \\
& \lesssim 4 \epsilon T+\sum_{i=0}^{\lfloor T / C \epsilon\rfloor} \int_{t_{i}}^{t_{i+1}} \frac{1}{N} \sum_{k=\lceil\epsilon N\rceil}^{N-\lfloor\epsilon N\rfloor}\left|H^{ \pm}\left(\tau_{k} \eta(t), \tau_{k} \zeta(t)\right)-H^{ \pm}\left(\tau_{k} \hat{\eta}(t), \tau_{k} \zeta(t)\right)\right| d t .
\end{aligned}
$$

To bound the second term on the right hand side, we first notice that there exists $K>0$ such that for any particle configurations $\eta_{1}, \eta_{2}, \eta_{1}^{\prime}$ and $\eta_{2}^{\prime}$, we have

$$
\left|H^{ \pm}\left(\eta_{1}, \eta_{2}\right)-H^{ \pm}\left(\eta_{1}^{\prime}, \eta_{2}^{\prime}\right)\right| \leqslant K \sum_{j=0,1} \sum_{m=1,2}\left|\eta_{m}(j)-\eta_{m}^{\prime}(j)\right|
$$


Consequently, Lemma 18 ensures that

$$
\begin{aligned}
\lim _{\ell \rightarrow \infty} \lim _{N \rightarrow \infty} \sum_{i=0}^{\lfloor T / \epsilon\rfloor} \mathbb{Q}^{N}\left[\int_{t_{i}}^{t_{i+1}} \frac{1}{N} \sum_{k=\lceil\epsilon N\rceil}^{N-\lfloor\epsilon N\rfloor} \mid H^{ \pm}\left(\tau_{k} \eta(t), \tau_{k} \zeta(t)\right)\right. \\
\left.-H^{ \pm}\left(\tau_{k} \hat{\eta}(t), \tau_{k} \zeta(t)\right) \mid d t\right]=0 .
\end{aligned}
$$

The same argument allows to control the second term in (45). Regarding the fourth term, it suffices to use the Lipschitz continuity of $h^{ \pm}$and Lemma 18. This concludes the proof.

Proof of Theorem 5. The two-blocks estimate [29, Lemma 6.6] ensures that one can replace averages on boxes of size $\ell$ by averages on boxes of size $\epsilon N$. Therefore, we deduce that the conclusion of Lemma 19 still holds upon such a replacement. Finally, one relies on classical arguments to show that this is sufficient to get the entropy inequalities, we refer the interested reader to [18, Proof of Th 2.7] for the details.

6. Locating the leftmost particle using the hydrodynamic profile. In this section we explain how Propositions 9 and 13 can be deduced from the hydrodynamic limit of the height function. The case $\alpha=0$ is a bit particular as, in that case, the limit of the height function is trivial under the scaling we consider. We tackle this case separately in Subsection 6.2

6.1. Particles performing ASEP on an infinite line. In order to complete the proof of Propositions 9 and 13, we combine Corollary 8 with a result that controls the speed of particles in sparse regions: the purpose of the present section is to expose the latter result.

We consider $n$ particles performing an ASEP on the infinite line $\mathbb{Z}$ with asymmetry $(p, q), n \in \llbracket 1, N \rrbracket$ and we want to obtain a lower bound on the displacement of the leftmost particle. We perform this operation in two steps: first we prove a concentration result and then we estimate the mean via stochastic comparison with a system in the stationary state.

In this section, instead of using zeros and ones to denote presence and absence of particles, we work with the state-space

$$
\Omega_{n}:=\left\{\hat{\eta}=\left(\hat{\eta}_{1}, \ldots, \hat{\eta}_{n}\right) \in \mathbb{Z}^{n}: \hat{\eta}_{1}<\hat{\eta}_{2}<\cdots<\hat{\eta}_{n}\right\} .
$$

With this notation, $\hat{\eta}_{i}$ denotes the position of the $i$-th particle starting from the left. For the sake of using stochastic comparisons we introduce the order

$$
\begin{gathered}
\hat{\eta} \leq \hat{\eta}^{\prime} \quad \Leftrightarrow \quad \forall i \in \llbracket 1, n \rrbracket, \hat{\eta}_{i} \leq \hat{\eta}_{i}^{\prime} . \\
\text { imsart-aop ver. 2014/10/16 file: AOP1290.tex date: May 9, } 2019
\end{gathered}
$$


Note that this order is the opposite of the order introduced in (16) at the level of height functions, but we believe that this will not raise any confusion.

We let $\hat{\eta}(t):=\left(\hat{\eta}_{1}(t), \ldots, \hat{\eta}_{n}(t)\right)$ denote the ASEP on $\mathbb{Z}$ with jump rates $p$ to the right and $q$ to the left, and with the initial condition $\hat{\eta}_{i}(0)=i$. Its distribution is denoted by $\mathbb{P}$. Our aim is to show that on "large" time-scales (i.e. larger than $n$ ), the speed of all particles is equal to what it would be in the absence of the exclusion rule: namely, $p-q$. The following result is valid for any given sequence $\left(n_{N}\right)_{N \geq 1}$ satisfying $n_{N} \in \llbracket 1, N \rrbracket$.

Proposition 20. Given $K>0$ there exists a constant $C(p, K)$ such that with high probability

$\forall t \in[0, K N], \forall i \in \llbracket 1, n \rrbracket, \quad\left|\hat{\eta}_{i}(t)-(p-q) t\right| \leq C \sqrt{N} \max \left(\sqrt{n},(\log N)^{10}\right)$.

6.2. Proof of Proposition 9 case $\alpha=0$. We start with the case $\alpha=0$ because it is substantially easier.

In order to obtain an upper bound for $L_{N, k}$, it is sufficient to say that the position of the leftmost particle $\eta_{1}$ of our original system is stochastically dominated by a $(p, q)$-biased simple random walk on the segment (the other $k-1$ particles to the right only slow it down). For the latter process, it is simple to check that, properly rescaled, it converges to $\max (t, 1)$ as $N \rightarrow \infty$.

To obtain a lower bound, let us consider the ASEP $\eta$ on the segment and the ASEP $\hat{\eta}$ on the full line with $n=k$. Take $\hat{\eta}_{i}(0)=i$ for every $i \in \llbracket 1, k \rrbracket$. We can couple the two processes in a way that $\eta(s) \geq \hat{\eta}(s)$ until the time

$$
\tau:=\inf \left\{s \geq 0: \hat{\eta}_{k}(s)=N\right\} .
$$

Then for any fixed $t<1$ and for any $\varepsilon>0$, Proposition 20 (with $n=k$ ) implies that with high probability $\hat{\eta}_{k}(s)<N$ for all $s<(p-q)^{-1} N t$, and that

$$
\hat{\eta}_{1}\left(\frac{N t}{p-q}\right) \geq N(t-\varepsilon) .
$$

This implies that

$$
\eta_{1}\left(\frac{N t}{p-q}\right) \geq N(t-\varepsilon) .
$$

For $t \geq 1$, the argument is essentially the same: one simply has to shift to the left the initial condition for $\hat{\eta}$. Namely, we set $\hat{\eta}_{i}(0)=N(1-t-\varepsilon)+i$ for $\varepsilon>0$ small. Then, for all $s \in\left[0,(p-q)^{-1} N t\right]$, we have $\hat{\eta}_{k}(s)<N$ with high probability. Furthermore, $\hat{\eta}_{1}\left(\frac{N t}{p-q}\right) \geq N(1-\varepsilon)$ with high probability so that

$$
\eta_{1}\left(\frac{N t}{p-q}\right) \geq N(1-\varepsilon),
$$

and the lower bound follows. 
6.3. Proof of Proposition 9 case $\alpha>0$. Let us fix some time horizon $t$.

In the case $\alpha>0$, we only need a lower bound on $L_{N, k}$ as the upper bound is an easy consequence of Corollary 8. We fix $\delta>0$ small and set $n=\delta N<k$. We want to compare the first $n$ particles of $\eta(t)$ with an ASEP with $n$ particles on the infinite line $\hat{\eta}=\left(\hat{\eta}_{1}, \ldots, \hat{\eta}_{n}\right)$. If the initial conditions are ordered we can couple $\hat{\eta}$ with $\eta$ in such a way that

$$
\forall i \in \llbracket 1, n \rrbracket, \quad \hat{\eta}_{i}(s) \leq \eta_{i}(s)
$$

until the first time that $\hat{\eta}_{n}(s)=\eta_{n+1}(s)$.

Corollary 8 implies that with a probability tending to one we have

$$
\forall s \in[0, t], \quad \eta_{n+1}\left(\frac{N s}{p-q}\right) \geq N \ell_{\alpha}(s)
$$

To ensure that our coupling works until the final time $t$, we choose the initial condition for $\hat{\eta}$ to be much smaller than that of $\eta$. We set

$$
\hat{\eta}_{i}(0)=N\left(\ell_{\alpha}(t)-t-\varepsilon\right)+i .
$$

From Proposition 20 we have w.h.p. for all $i \in \llbracket 1, n \rrbracket$

$$
\forall s \in\left[0, \frac{N t}{p-q}\right], \quad\left|\hat{\eta}_{i}(s)-(p-q) s-N\left(\ell_{\alpha}(t)-t-\varepsilon\right)\right| \leq C \sqrt{\delta} N .
$$

Together with (48), and provided $\delta$ is sufficiently small given $\varepsilon$, this implies that the probability of the event

$$
\forall s \in\left[0, \frac{N t}{p-q}\right], \quad \hat{\eta}_{n}(s)<\eta_{n+1}(s),
$$

goes to 1 as $N \rightarrow \infty$. Thanks to (47), this implies in turn that w.h.p.

$$
\eta_{1}\left(\frac{N t}{p-q}\right) \geq \hat{\eta}_{1}\left(\frac{N t}{p-q}\right)
$$

and thus we deduce from (49) that

$$
\hat{\eta}_{1}\left(\frac{N t}{p-q}\right) \geq N\left(\ell_{\alpha}(t)-\varepsilon-C \sqrt{\delta}\right) .
$$

As both $\delta$ and $\varepsilon$ can be chosen arbitrarily small, this allows to conclude. 
6.4. Proof of Proposition 13. In that case, the system starts from $\xi_{N}$ instead of $\wedge$, so we need to adapt the arguments. We treat only the case where the limiting density $\alpha$ is strictly positive, the case $\alpha=0$ being simpler is left to the reader. Fix $t \geq 0$. The proof of the upper bound is simple. Either $\ell_{\rho}(t)>\ell+t$, and then it suffices to compare $L_{N}$ with a biased $(p, q)$ simple random walk as we did in Subsection 6.2. Or $\ell_{\rho}(t) \leqslant \ell+t$, and then the upper bound is a consequence of the hydrodynamic limit stated in Theorem 4. We turn to the proof of the lower bound. The arguments are essentially the same as those presented in Subsection 6.3, let us spell out the required modifications. The bound in (48) still holds if one replaces $\ell_{\alpha}(s)$ by $\ell_{\rho}(s)$. The initial condition has to be taken as follows:

$$
\hat{\eta}_{i}(0)=N\left(\left[(\ell+t) \wedge \ell_{\rho}(t)\right]-t-\varepsilon\right)+i .
$$

Since the speed of $\ell_{\rho}$ is necessarily bounded above by 1 , we deduce that w.h.p., $\hat{\eta}_{i}(0) \leq \eta_{i}(0)$ for all $i \in \llbracket 1, n \rrbracket$. Then, Proposition 20 ensures that

$\forall s \in\left[0, \frac{N t}{p-q}\right], \quad\left|\hat{\eta}_{i}(s)-(p-q) s-N\left((\ell+t) \wedge \ell_{\rho}(t)-t-\varepsilon\right)\right| \leq C \sqrt{\delta} N$.

The rest of the arguments then apply and we deduce that $\eta_{1}(t) \geq N((\ell+$ t) $\left.\wedge \ell_{\rho}(t)-\varepsilon-C \sqrt{\delta}\right)$ w.h.p., thus concluding the proof of Proposition 13 in the case $\alpha>0$.

6.5. Proof of Proposition 20. Since the system is ordered, we only need to prove that the following two inequalities hold w.h.p.

$$
\begin{array}{ll}
\forall t \in[0, K N], & \hat{\eta}_{1}(t) \geq(p-q) t-C \sqrt{N} \max \left(\sqrt{n},(\log N)^{10}\right), \\
\forall t \in[0, K N], & \hat{\eta}_{n}(t) \leq(p-q) t+C \sqrt{N} \max \left(\sqrt{n},(\log N)^{10}\right) .
\end{array}
$$

The proof of the second inequality is in fact very similar to the proof of the first one. Hence we decide to discuss in detail only the case of $\hat{\eta}_{1}$ and we explain briefly the needed modifications for $\hat{\eta}_{n}$ when they are non trivial.

The proof of the result is decomposed into two separate statements: first we show that $\hat{\eta}_{1}(n t)$ is concentrated around its mean using a martingale concentration result from [23], and then we obtain a lower bound on $\mathbb{E}\left[\hat{\eta}_{1}(n t)\right]$ by comparing the system with a stationary one.

LEMMA 21. Under the assumptions above, there exists $c>0$ such that for all $n \geqslant 2$, all $t \geq 0$, all $i \in \llbracket 1, n \rrbracket$ and all $u \geqslant 0$ such that $u^{2} /\left(t(\log n)^{2}\right)>$ 1, we have

$$
\begin{aligned}
& \mathbb{P}\left[\left|\hat{\eta}_{i}(t)-\mathbb{E}\left[\hat{\eta}_{i}(t)\right]\right| \geq u\right] \leq 2 \exp \left(-c\left(\frac{u^{2}}{t(\log n)^{2}}\right)^{1 / 3}\right) \\
& \quad \text { imsart-aop ver. } 2014 / 10 / 16 \text { file: AOP1290.tex date: May } 9,2019
\end{aligned}
$$


LEMMA 22. With the assumptions above we have

$$
\begin{aligned}
& \mathbb{E}\left[\hat{\eta}_{1}(t)\right] \geq(p-q) t-2 \max (n, \sqrt{t n}), \\
& \mathbb{E}\left[\hat{\eta}_{n}(t)\right] \leq(p-q) t+2 \max (n, \sqrt{t n}) .
\end{aligned}
$$

We postpone the proofs of the lemmas to the end of the subsection and we proceed to the proof of (50). Combining the two lemmas, we obtain easily that for any $t \in[0, K N]$ we have

$$
\mathbb{P}\left[\hat{\eta}_{1}(t) \leq(p-q) t-C \sqrt{N} \max \left(\sqrt{n},(\log N)^{4}\right)\right] \leq \exp \left(-c(\log N)^{2}\right),
$$

for some constants $c, C>0$. Hence, the probability that there exists $t \in$ $\left\{j N^{-4}, j \in \llbracket 1, K N^{5} \rrbracket\right\}$ such that

$$
\hat{\eta}_{1}(t) \leq(p-q) t-C \sqrt{N} \max \left(\sqrt{n},(\log N)^{4}\right),
$$

is bounded by $K N^{5} \exp \left(-c(\log N)^{2}\right)$ which vanishes as $N \rightarrow \infty$. Then, we notice that the probability that there exists an interval $\left[j N^{-4},(j+1) N^{-4}\right)$ on which $\hat{\eta}_{1}$ makes more than one jump is bounded by a term of order $N^{5}$ times the probability that a Poisson clock rings more than once in a time interval of length $N^{-4}$, that is, by a term of order $N^{-3}$. We deduce that the probability that there exists $t \in\left[0, K N^{5}\right]$ such that

$$
\hat{\eta}_{1}(t) \leq(p-q) t-C \sqrt{N} \max \left(\sqrt{n},(\log N)^{4}\right)-1,
$$

is vanishing with $N$, from which we deduce (50) for $\hat{\eta}_{1}$. A very similar argument yields (50) for $\hat{\eta}_{n}$.

Proof of Lemma 21. First, let us consider the case where $t$ is an integer. Fix $i \in \llbracket 1, n \rrbracket$. For such a $t$, we define the martingale $\left(M_{s}^{t}, s \in \llbracket 0, t \rrbracket\right)$ by

$$
M_{s}^{t}:=\mathbb{E}\left[\hat{\eta}_{i}(t) \mid \mathcal{F}_{s}\right]-\mathbb{E}\left[\hat{\eta}_{i}(t)\right], \quad \mathcal{F}_{s}:=\sigma(\hat{\eta}(u), u \in[0, s]) .
$$

We are going to prove tail bounds on the increments of $M^{t}$ to obtain concentration. For convenience, we set $\Delta M_{s}^{t}:=M_{s}^{t}-M_{s-1}^{t}$ for any $s \in \llbracket 1, t \rrbracket$. We let $\bar{R}(s)$, resp. $\bar{L}(s)$, denote the maximal number of jumps to the right, resp. left, performed by a particle in the system during the time interval $(s-1, s]$,

$$
\begin{array}{r}
\bar{R}(s):=\max _{i \in \llbracket 1, n \rrbracket} \#\left\{t \in(s-1, s]: \hat{\eta}_{i}(t)=\hat{\eta}_{i}\left(t_{-}\right)+1\right\}=\max _{i \in \llbracket 1, n \rrbracket} R_{i}(s), \\
\bar{L}(s):=\max _{i \in \llbracket 1, n \rrbracket} \#\left\{t \in(s-1, s]: \hat{\eta}_{i}(t)=\hat{\eta}_{i}\left(t_{-}\right)-1\right\}=\max _{i \in \llbracket 1, n \rrbracket} L_{i}(s) . \\
\quad \text { imsart-aop ver. 2014/10/16 file: AOP1290.tex date: May 9, } 2019
\end{array}
$$


At time $s$ we have

$$
\hat{\eta}(s-1)-\bar{L}(s) \leq \hat{\eta}(s) \leq \hat{\eta}(s-1)+\bar{R}(s) .
$$

where for $k \in \mathbb{N}$ and $\hat{\eta} \in \Omega_{n}$,

$$
\hat{\eta}+k:=\left(\hat{\eta}_{1}+k, \ldots, \hat{\eta}_{n}+k\right) .
$$

Let us now consider two initial conditions given by $\hat{\eta}(s-1)$ on the one hand, and $\hat{\eta}(s)+\bar{L}(s)$ on the other hand, and let us run the ASEP dynamics for both systems for a time length $t-s$ : the two configurations are stochastically ordered, and their laws coincide with the laws of $\hat{\eta}(t-1)$ conditionally given $\mathcal{F}_{s-1}$ for the first one and of $\hat{\eta}(t)+\bar{L}(s)$ conditionally given $\mathcal{F}_{s}$ for the second one. A similar reasoning can be applied to $\hat{\eta}(s-1)$ and $\hat{\eta}(s)+\bar{R}(s)$. Therefore, we get for all $t \geqslant s$

$$
\begin{aligned}
& \mathbb{E}\left[\hat{\eta}_{i}(t) \mid \mathcal{F}_{s}\right] \leq \mathbb{E}\left[\hat{\eta}_{i}(t-1) \mid \mathcal{F}_{s-1}\right]+\bar{R}(s), \\
& \mathbb{E}\left[\hat{\eta}_{i}(t) \mid \mathcal{F}_{s}\right] \geq \mathbb{E}\left[\hat{\eta}_{i}(t-1) \mid \mathcal{F}_{s-1}\right]-\bar{L}(s) .
\end{aligned}
$$

Furthermore as we have $-L_{i}(t) \leq \hat{\eta}_{i}(t)-\hat{\eta}_{i}(t-1) \leq R_{i}(t)$, and as both variables $R_{i}(t)$ and $L_{i}(t)$ are, conditionally to $\mathcal{F}_{t-1} \supset \mathcal{F}_{s-1}$, dominated by Poisson random variables of parameters $p$ and $q$, we have for any $s \leq t$,

$$
\left|\mathbb{E}\left[\hat{\eta}_{i}(t) \mid \mathcal{F}_{s-1}\right]-\mathbb{E}\left[\hat{\eta}_{i}(t-1) \mid \mathcal{F}_{s-1}\right]\right| \leq \max (p, q)=p .
$$

Then, we write

$$
\begin{aligned}
\left|\Delta M_{s}^{t}\right| \leq & \left|\mathbb{E}\left[\hat{\eta}_{i}(t) \mid \mathcal{F}_{s-1}\right]-\mathbb{E}\left[\hat{\eta}_{i}(t-1) \mid \mathcal{F}_{s-1}\right]\right| \\
& +\left|\mathbb{E}\left[\hat{\eta}_{i}(t) \mid \mathcal{F}_{s}\right]-\mathbb{E}\left[\hat{\eta}_{i}(t-1) \mid \mathcal{F}_{s-1}\right]\right| \\
\leq & p+\max (\bar{R}(s), \bar{L}(s)) .
\end{aligned}
$$

As $\bar{R}(s)$ and $\bar{L}(s)$ are bounded above by the maxima of $n$ Poisson variables of mean $p$ and $q$, we obtain that for any constant $c_{p}>0$ there exists $C>0$ such that

$$
\mathbb{P}\left[\left|\Delta M_{s}^{t}\right| \geq u\right] \leq C n e^{-c_{p} u}, \quad \forall u \geqslant 0 .
$$

This implies the existence of $K>0$, independent of $n$, such that

$$
\mathbb{E}\left[e^{(\log n)^{-1} \Delta M_{s}^{t}}\right] \leq K
$$

Hence, we can apply [23, Theorem 3.2] (which is simply Azuma's inequality combined with some truncation argument for the increments) to the martingale $(\log n)^{-1} M_{s}^{t}$. More precisely, we apply the bound obtained at the end 
of the proof of Theorem 3.2 therein (equation right below (11)) and deduce that the asserted concentration estimate holds.

So far, we have proven the bound when $t \in \mathbb{N}$. To treat the general case $t \geqslant 0$, it suffices to bound the increment $M_{t}^{t}-M_{\lfloor t\rfloor}^{t}$. Inspecting the arguments above, we observe that (51) still holds in that case, so that the proof carries through.

Proof of Lemma 22. We notice that adding particles to the right, reducing the drift of some particles, or changing the initial condition by shifting the particles to the left have the effect of slowing down $\hat{\eta}_{1}(t)$ in the sense that the system obtained after such modifications is dominated by the original one.

We consider more specifically the following modification of the dynamics which we call $\tilde{\eta}$ :

- $\tilde{\eta}_{n}(0)=n$ and $\left(\tilde{\eta}_{i+1}-\tilde{\eta}_{i}\right)_{i=1, \ldots, n-1}$ are IID geometric random variables of parameter $\mu<1$, that is, $P\left[\tilde{\eta}_{i+1}-\tilde{\eta}_{i}=k\right]=(1-\mu) \mu^{k-1}$,

- The jump rate to the left is still $q$ but the jump rate to the right is $p$ for the first (leftmost) particle, $p_{1}$ for particles labeled from 2 to $n-1$ and $p_{2}$ for the last (rightmost) one.

It can be checked that the product of geometric laws with parameter $\mu$ is stationary (but not reversible in general) for the Markov chain $\left[\left(\tilde{\eta}_{i+1}(t)-\tilde{\eta}_{i}(t)\right)_{i=1}^{n-1}\right]_{t \geq 0}$, provided that

$$
\mu(p+q)=\mu p_{1}+q=p_{2}+q .
$$

Note that this implies $p_{2} \leq p_{1} \leq p$, and therefore as we have $\hat{\eta}(0) \geq \tilde{\eta}(0)$ this implies that there exists a coupling such that

$$
\forall t \geq 0, \quad \hat{\eta}(t) \geq \tilde{\eta}(t)
$$

As the increments are stationary, the expected drift of the first particle is the same as the initial drift and thus

$$
\forall t \geq 0, \partial_{t} \mathbb{E}\left[\tilde{\eta}_{1}(t)\right]=p \mu-q
$$

Moreover

$$
\mathbb{E}\left[\tilde{\eta}_{1}(0)\right]=1-(n-1) \frac{\mu}{1-\mu} .
$$

Using (52) this implies that

$$
\mathbb{E}\left[\hat{\eta}_{1}(t)\right] \geq 1+(p \mu-q) t-\frac{\mu(n-1)}{1-\mu} \geq(p-q) t-(1-\mu) t-\frac{n}{1-\mu} .
$$


Choosing $\mu$ such that $1-\mu=\min (1, \sqrt{n / t})$ we obtain

$$
\mathbb{E}\left[\hat{\eta}_{1}(t)\right] \geq(p-q) t-2 \max (n, \sqrt{n t}),
$$

as required.

To establish the asserted result for the rightmost particle, we consider an analogous system where particle spacings have the same initial distribution (IID geometric random variables with parameter $\mu$ ), but we fix $\tilde{\eta}_{1}(0)=1$ and set the jump rate to the right to be $p_{4}$ for the first particle, $p_{3}$ for the particles with labels from 2 to $n-1$ and $p$ for the particle with label $n$, where

$$
\mu\left(p_{4}+q\right)=\mu p_{3}+q=p+q .
$$

We can couple $\hat{\eta}$ and $\tilde{\eta}$ in a way such that $\tilde{\eta}(t) \geq \hat{\eta}(t)$ for all $t \geqslant 0$. The speed of the rightmost particle is given by $p-\mu q$, and its initial mean is $1+(n-1) \frac{1}{1-\mu}$. Taking $1-\mu=\min (1, \sqrt{n / t})$ yields the bound

$$
\mathbb{E}\left[\hat{\eta}_{n}(t)\right] \leq(p-q) t+2 \max (n, \sqrt{n t})
$$

as required.

Acknowledgements: The present work was partially realized during a visit of H.L. to CEREMADE, he acknowledges kind hospitality and support. H.L. also acknowledges the support of a productivity grant from CNPq.

\section{APPENDIX A: GRAND COUPLING}

We are given two collections $\left(P_{i}, i \in \llbracket 1, N-1 \rrbracket\right)$ and $\left(Q_{i}, i \in \llbracket 1, N-1 \rrbracket\right)$ of independent Poisson processes with jump rates $p$ and $q$ respectively. The grand coupling for the biased card shuffling is defined as follows. For any initial condition $\xi \in \mathcal{S}_{N}$, the process $\sigma^{\xi}$ starts from $\sigma_{0}^{\xi}=\xi$ and is piecewise constant outside of the jump times of $P_{i}$ and $Q_{i}$. The transition at these latter time are defined as follows: At every jump time $s>0$ of $P_{i}$ we place the cards at sites $i, i+1$ in the increasing order, that is, $\sigma_{s}^{\xi}=\sigma_{s-}^{\xi} \circ \tau_{i}$ if $\sigma_{s-}^{\xi}(i)>\sigma_{s-}^{\xi}(i+1)$ and $\sigma_{s}^{\xi}=\sigma_{s-}^{\xi}$ otherwise. At every jump time $s>0$ of $Q_{i}$ we place the cards at sites $i, i+1$ in the decreasing order, that is, $\sigma_{s}^{\xi}=\sigma_{s-}^{\xi} \circ \tau_{i}$ if $\sigma_{s-}^{\xi}(i)<\sigma_{s-}^{\xi}(i+1)$ and $\sigma_{s}^{\xi}=\sigma_{s-}^{\xi}$ otherwise.

Taking the image of this process through the maps $h_{k}: \mathcal{S}_{N} \rightarrow \Omega_{N, k}$ for $k \in \llbracket 1, N-1 \rrbracket$, we get a grand coupling of the asymmetric simple exclusion processes. The dynamics at the level of the height functions can be restated as follows: at a jump time of $P_{i}$, if the height function makes an upwards 
corner at site $i$ then it flips into a downwards corner; similarly, at a jump time of $Q_{i}$, if the height function makes a downwards corner at site $i$ then it flips into an upwards corner.

Let us check that the dynamics preserves the order. To that end, it suffices to check that all the transitions do so. Consider a jump time of $P_{i}$ and suppose we are given two heights functions $h \leqslant h^{\prime}$ right before the jump time. If both $h$ and $h^{\prime}$ (or none of them) have an upwards corner at site $i$, then both flip downwards and the ordering is preserved. If only $h$ has an upwards corner, then the flip can only make $h$ smaller and therefore the ordering is also preserved upon the jump. Let us now suppose that only $h^{\prime}$ has an upwards corner at site $i$. Inspecting the possible shapes for $h$ at site $i$, and recalling that the set of possible values for the height function at a given site $i$ has a span equal to 2 we deduce that necessarily $h(i) \leqslant h^{\prime}(i)+2$. Therefore, after the jump the ordering is still preserved at site $i$. By symmetry, the arguments are the same for upwards flips. This concludes the proof.

\section{REFERENCES}

[1] Bahadoran, C. (2012). Hydrodynamics and hydrostatics for a class of asymmetric particle systems with open boundaries. Comm. Math. Phys. 310 1-24. MR2885612

[2] Bardos, C., le Roux, A. Y. and Nédélec, J. C. (1979). First order quasilinear equations with boundary conditions. Comm. Partial Differential Equations 4 10171034. MR542510 (81b:35052)

[3] Benassi, A. and Fouque, J.-P. (1987). Hydrodynamical limit for the asymmetric simple exclusion process. Ann. Probab. 15 546-560. MR885130

[4] Benjamini, I., Berger, N., Hoffman, C. and Mossel, E. (2005). Mixing times of the biased card shuffling and the asymmetric exclusion process. Trans. Amer. Math. Soc. 357 3013-3029 (electronic). MR2135733

[5] Bertini, L. and Giacomin, G. (1997). Stochastic Burgers and KPZ equations from particle systems. Comm. Math. Phys. 183 571-607. MR1462228 (99e:60212)

[6] Bhakta, P., Miracle, S., Randall, D. and Streib, A. P. (2012). Mixing times of Markov chains for self-organizing lists and biased permutations. In Proceedings of the Twenty-Fourth Annual ACM-SIAM Symposium on Discrete Algorithms 1-15. SIAM, Philadelphia, PA. MR3185376

[7] Billingsley, P. (1999). Convergence of probability measures, second ed. Wiley Series in Probability and Statistics: Probability and Statistics. John Wiley \& Sons, Inc., New York A Wiley-Interscience Publication. MR1700749 (2000e:60008)

[8] Bürger, R., Frid, H. and Karlsen, K. H. (2007). On the well-posedness of entropy solutions to conservation laws with a zero-flux boundary condition. J. Math. Anal. Appl. 326 108-120. MR2277770

[9] Caputo, P., Liggett, T. M. and Richthammer, T. (2010). Proof of Aldous' spectral gap conjecture. J. Amer. Math. Soc. 23 831-851. MR2629990

[10] Caputo, P., Lacoin, H., Martinelli, F., Simenhaus, F. and Toninelli, F. L. (2012). Polymer dynamics in the depinned phase: metastability with logarithmic barriers. Probab. Theory Related Fields 153 587-641. MR2948687

[11] Derrida, B. and Lebowitz, J. L. (1998). Exact Large Deviation Function in the Asymmetric Exclusion Process. Phys. Rev. Lett. 80 209-213.

imsart-aop ver. 2014/10/16 file: AOP1290.tex date: May 9, 2019 
[12] Diaconis, P. and Shahshahani, M. (1987). Time to reach stationarity in the Bernoulli-Laplace diffusion model. SIAM J. Math. Anal. 18 208-218. MR871832

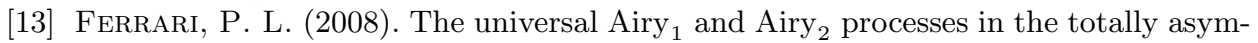
metric simple exclusion process. In Integrable systems and random matrices. Contemp. Math. 458 321-332. Amer. Math. Soc., Providence, RI. MR2411915

[14] GärTner, J. (1988). Convergence towards Burgers' equation and propagation of chaos for weakly asymmetric exclusion processes. Stochastic Process. Appl. $27233-$ 260. MR931030 (89e:60200)

[15] Greenberg, S., Pascoe, A. and Randall, D. (2009). Sampling biased lattice configurations using exponential metrics. In Proceedings of the Twentieth Annual ACM-SIAM Symposium on Discrete Algorithms 76-85. SIAM, Philadelphia, PA. MR2809307

[16] Haddadan, S. and Winkler, P. (2016). Mixing of Permutations by Biased Transposition. ArXiv e-prints.

[17] Kipnis, C. and LAndim, C. (1999). Scaling limits of interacting particle systems. Grundlehren der Mathematischen Wissenschaften [Fundamental Principles of Mathematical Sciences] 320. Springer-Verlag, Berlin. MR1707314 (2000i:60001)

[18] LABBÉ, C. (2017). Weakly asymmetric bridges and the KPZ equation. Comm. Math. Phys. 353 1261-1298. MR3652491

[19] Labbé, C. and Lacoin, H. Mixing time and cutoff for the Weakly Asymmetric Simple Exclusion Process. In preparation.

[20] Lacoin, H. (2016a). Mixing time and cutoff for the adjacent transposition shuffle and the simple exclusion. Ann. Probab. 44 1426-1487. MR3474475

[21] LaCOIN, H. (2016b). The cutoff profile for the simple exclusion process on the circle. Ann. Probab. 44 3399-3430. MR3551201

[22] LEe, T.-Y. and YAU, H.-T. (1998). Logarithmic Sobolev inequality for some models of random walks. Ann. Probab. 26 1855-1873. MR1675008

[23] Lesigne, E. and Volný, D. (2001). Large deviations for martingales. Stochastic Process. Appl. 96 143-159. MR1856684

[24] Levin, D. A., Peres, Y. and Wilmer, E. L. (2009). Markov chains and mixing times. Providence, R.I. American Mathematical Society With a chapter on coupling from the past by James G. Propp and David B. Wilson.

[25] Levin, D. A. and Peres, Y. (2016). Mixing of the exclusion process with small bias. ArXiv e-prints.

[26] Liggett, T. M. (2005). Interacting particle systems. Classics in Mathematics. Springer-Verlag, Berlin Reprint of the 1985 original. MR2108619

[27] Málek, J., NeČAs, J., Rokyta, M. and RuŽıČKA, M. (1996). Weak and measurevalued solutions to evolutionary PDEs. Applied Mathematics and Mathematical Computation 13. Chapman \& Hall, London. MR1409366

[28] Отто, F. (1996). Initial-boundary value problem for a scalar conservation law. $C$. R. Acad. Sci. Paris Sér. I Math. 322 729-734. MR1387428

[29] Rezakhanlou, F. (1991). Hydrodynamic limit for attractive particle systems on $\mathbf{Z}^{d}$. Comm. Math. Phys. 140 417-448. MR1130693 (93f:82035)

[30] Rost, H. (1981). Nonequilibrium behaviour of a many particle process: density profile and local equilibria. Z. Wahrsch. Verw. Gebiete 58 41-53. MR635270

[31] Vovelle, J. (2002). Convergence of finite volume monotone schemes for scalar conservation laws on bounded domains. Numer. Math. 90 563-596. MR1884231 (2002k:65158)

[32] Wilson, D. B. (2004). Mixing times of Lozenge tiling and card shuffling Markov chains. Ann. Appl. Probab. 14 274-325. MR2023023

imsart-aop ver. 2014/10/16 file: AOP1290.tex date: May 9, 2019 
PSL Research University, Ceremade, 75775 Paris Cedex 16, France. E-mail: labbe@ceremade.dauphine.fr impa, Estrada Dona Castorina 110, Rio de Janeiro, Brasil. E-mail: lacoin@impa.br 